\title{
Ramification in the division fields of elliptic curves with potential supersingular reduction
}

Álvaro Lozano-Robledo

Correspondence:

alvaro.lozano-robledo@uconn.edu Department of Mathematics,

University of Connecticut, Storrs, CT 06269, USA

\section{照 Springer}

\begin{abstract}
Let $d \geq 1$ be fixed. Let $F$ be a number field of degree $d$, and let $E / F$ be an elliptic curve. Let $E(F)_{\text {tors }}$ be the torsion subgroup of $E(F)$. In 1996, Merel proved the uniform boundedness conjecture, i.e., there is a constant $B(d)$, which depends on $d$ but not on the chosen field $F$ or on the curve $E / F$, such that the size of $E(F)_{\text {tors }}$ is bounded by $B(d)$. Moreover, Merel gave a bound (exponential in $d$ ) for the largest prime that may be a divisor of the order of $E(F)_{\text {tors. }}$ In 1996, Parent proved a bound (also exponential in $d$ ) for the largest $p$-power order of a torsion point that may appear in $E(F)_{\text {tors. }}$. It has been conjectured, however, that there is a bound for the size of $E(F)_{\text {tors }}$ that is polynomial in $d$. In this article we show that if $E / F$ has potential supersingular reduction at a prime ideal above $p$, then there is a linear bound for the largest $p$-power order of a torsion point defined over $F$, which in fact is linear in the ramification index of the prime of supersingular reduction.
\end{abstract}

1991 Mathematics Subject Classification: Primary: 11G05, Secondary: 14H52

\section{Introduction}

Let $F$ be a number field, and let $E / F$ be an elliptic curve defined over $F$. The Mordell-Weil theorem states that $E(F)$, the set of $F$-rational points on $E$, can be given the structure of a finitely generated abelian group. In particular, the torsion subgroup of $E(F)$, henceforth denoted by $E(F)_{\text {tors }}$, is a finite group. In 1996, Merel proved that there is a uniform bound for the size of $E(F)_{\text {tors }}$, which is independent of the chosen curve $E / F$ and, in fact, the bound only depends on the degree of $F / \mathbb{Q}$. The bounds were improved by Oesterlé, and later by Parent.

Theorem 1.1 (Merel, [7], and Parent, [9]). Let $p$ be a prime, let $d>1$ be a fixed integer, let $F$ be a number field $F$ of degree $\leq d$ and let $E / F$ be an elliptic curve. Then:

- (Oesterlé, 1996) If $E(F)$ contains a point of exact order $p$, then $p \leq\left(1+3^{d / 2}\right)^{2}$.

- (Parent, 1999) If $E(F)$ contains a point of exact order $p^{n}$, then $p^{n} \leq 129\left(5^{d}-1\right)(3 d)^{6}$.

In this article, we study the ramification index in the field of definition of $p^{n}$-th torsion points. Let $L$ be a number field, let $p$ be a prime, let $n \geq 1$, and let $\zeta=\zeta_{p^{n}}$ be a primitive $p^{n}$-th root of unity. Let $\wp$ be a prime ideal of the ring of integers $\mathcal{O}_{L}$

(c) 2016 Lozano-Robledo. Open Access This article is distributed under the terms of the Creative Commons Attribution 4.0 International License (http://creativecommons.org/licenses/by/4.0/), which permits unrestricted use, distribution, and reproduction in any medium, provided you give appropriate credit to the original author(s) and the source, provide a link to the Creative Commons license, and indicate if changes were made. 
of $L$ lying above $p$. The ramification index of the primes above $\wp$ in the extension $L(\zeta) / L$ is a divisor of $\varphi\left(p^{n}\right)$, where $\varphi(\cdot)$ is the Euler phi function, and, in fact, it is easy to see that the index is divisible by $\varphi\left(p^{n}\right) / \operatorname{gcd}\left(\varphi\left(p^{n}\right), e(\wp \mid p)\right)$. In this article we study the ramification above $p$ in the extension $L(R) / L$, where $R$ is a torsion point of exact order $p^{n}$ in an elliptic curve $E$ defined over $L$. In particular, we concentrate on the case when $E / L$ has potential good supersingular reduction at $\wp$. We show the following:

Theorem 1.2. Let $n \geq 1$ be fixed. Let $p$ be a prime, let $L$ be a number field, and let $\wp$ be a prime ideal of $\mathcal{O}_{L}$ lying above $p$. Let $E / L$ be an elliptic curve with potential supersingular reduction at $\wp$, let $R \in E\left[p^{n}\right]$ be a point of exact order $p^{n}$. Then, there is a computable constant $c=c(E / L, R, \wp)$ with $1 \leq c \leq 24 e(\wp \mid p)$ (with $c \leq 12 e(\wp \mid p)$ if $p>2$, and $c \leq 6 e(\wp \mid p)$ if $p>3)$, such that the ramification index $e(\mathfrak{P} \mid \wp)$ of any prime $\mathfrak{P}$ above $\wp$ in the extension $L(R) / L$ is divisible by

$$
\varphi\left(p^{n}\right) / \operatorname{gcd}\left(\varphi\left(p^{n}\right), c(E / L, R, \wp)\right) .
$$

Moreover:

1. For each $\eta \geq 1$, there is a constant $f(\eta)$ such that if $L$ is a any number field with $e(\wp \mid p) \leq \eta$, and $E / L$ and $R$ are as above, then $e(\mathfrak{P} \mid \wp)$ is divisible by $\varphi\left(p^{n}\right) / \operatorname{gcd}\left(\varphi\left(p^{n}\right), f(\eta)\right)$.

2. If $e(\wp \mid p)=1$ and $p>3$, then $e(\mathfrak{P} \mid \wp)$ is divisible by either $\left(p^{2}-1\right) p^{2(n-1)} / 6$, or the quantity $(p-1) p^{2(n-1)} / \operatorname{gcd}(p-1,4)$.

Theorem 1.2 is shown by providing a complete description and exact formulas of the slopes of the formal group of $E / L$ (see Corollary 4.2). These formulas lead to exact formulas for the valuation of the roots of the formal group (see Lemma 5.3), which in turn lead to exact formulas for the ramification indices above $p$ of the extension $L(R) / L$ when $R \in E\left[p^{n}\right]$ is a point of exact order $p^{n}$ (see Proposition 5.6). For instance, if $E / \mathbb{Q}: y^{2}+y=x^{3}-30 x+63$, and if $R_{n} \in E(\overline{\mathbb{Q}})$ is a point of exact order $3^{n}$ with $n \geq 3$, then the ramification index of any prime lying above 3 in the extension $\mathbb{Q}\left(R_{n}\right) / \mathbb{Q}$ is divisible by $3^{2 n-4}$, and that is precisely the ramification index for certain choices of $R_{n}$ (see Example 5.10). Similarly, if $E / \mathbb{Q}$ is the curve with Cremona label $121 c 2$, and $R_{n} \in E(\overline{\mathbb{Q}})$ is a point of exact order $11^{n}$ with $n \geq 1$, then the ramification index of any prime lying above 11 in the extension $\mathbb{Q}\left(R_{n}\right) / \mathbb{Q}$ is divisible by $5 \cdot 11^{2(n-1)}$, and this is again best possible (see Example 5.11).

Moreover, under the assumptions of Theorem 1.2 we have

$$
[L(R): L] \geq e(\mathfrak{P} \mid \wp) \geq \frac{\varphi\left(p^{n}\right)}{\operatorname{gcd}\left(\varphi\left(p^{n}\right), c(E / L, R, \wp)\right)} \geq \frac{\varphi\left(p^{n}\right)}{24 e(\wp \mid p)},
$$

and therefore

$$
\varphi\left(p^{n}\right) \leq 24 e(\wp \mid p) e(\mathfrak{P} \mid \wp)=24 e(\mathfrak{P} \mid p) \leq 24 \cdot[L(R): L] .
$$

Hence, as a consequence of our main Theorem 1.2, we show a similar bound to Theorem 1.1 in the supersingular reduction case, which is linear in $d$ (instead of exponential as in Theorem 1.1) and, in fact, it only depends on the ramification index of a prime of $F$ above $p$. 
Theorem 1.3. Let $p$ be a prime, let $d \geq 1$ be a fixed integer, let $F$ be a number field of degree $\leq d$, and let $E / F$ be an elliptic curve, such that $E(F)$ contains a point of exact order $p^{n}$. Suppose that $F$ has a prime $\mathfrak{P}$ over $p$ such that $E / F$ has potential good supersingular reduction at $\mathfrak{P}$. Then,

$$
\varphi\left(p^{n}\right) \leq\left\{\begin{array}{l}
24 e(\mathfrak{P} \mid p) \leq 24 d \text { if } p=2 \\
12 e(\mathfrak{P} \mid p) \leq 12 d \text { if } p=3, \\
6 e(\mathfrak{P} \mid p) \leq 6 d \quad \text { if } p>3
\end{array}\right.
$$

and $e(\mathfrak{P} \mid p)$ is the ramification index of $\mathfrak{P}$ in $F / \mathbb{Q}$.

Thus, Theorem 1.2 when applied uniformly recovers bounds previously found by Flexor and Oesterlé, who show $\left|E(F)_{\text {tors }}\right| \leq 48 d$ under similar hypotheses (see [2], Théorème 2). Our results, however, emphasize that there is a bound which is linear with respect to a ramification index of $F / \mathbb{Q}$, and can be regarded as evidence towards the following conjecture of the author, which will be discussed more in depth in an upcoming article.

Conjecture 1.4 ([6]). Let $p$ be a prime, let $d>1$ be a fixed integer, let $F$ be a number field of degree $\leq d$, and let $E / F$ be an elliptic curve, such that $E(F)$ contains a point of exact order $p^{n}$. There is a constant $C_{3}$ that does not depend on $p, d, F$ or $E$, such that

$$
\varphi\left(p^{n}\right) \leq C_{3} \cdot e_{\max }(p, F / \mathbb{Q}) \leq C_{3} \cdot d,
$$

where $e_{\max }(p, F / \mathbb{Q})$ is the largest ramification index $e(\mathfrak{P} \mid p)$ for a prime $\mathfrak{P}$ of $\mathcal{O}_{F}$ over the rational prime $p$.

The paper is organized as follows. In Section 2 we discuss generalities about elliptic curves with potential good reduction, concentrating on the potential supersingular reduction case. In Section 3 we summarize results on the formal group of elliptic curves with potential supersingular reduction from [4], which we generalize in Section 4. In Section 5, we use these results to study the $p$-adic valuation of $p^{n}$-th torsion points, and the ramification index of the extensions generated by torsion points. It is here that we show Theorem 5.9, which subsumes Theorem 1.2. Throughout the paper, we exemplify our results with the elliptic curves $E_{27 \mathrm{a} 4} / \mathbb{Q}$ and $E_{121 \mathrm{c} 2} / \mathbb{Q}$ with Cremona labels "27a4" and "121c2", and the primes $p=3$ and 11, respectively. In the last section of the article, Section 6, we discuss several other examples that correspond to non-cuspidal rational points on the modular curves $X_{0}\left(p^{n}\right)$, which appear in applications such as [5], and also we work out an example with an elliptic curve defined over a (quadratic) number field (see Example 6.2).

\section{Potential good reduction}

Let $L$ be a number field with ring of integers $\mathcal{O}_{L}$, let $p \geq 2$ be a prime, let $\wp$ be a prime ideal of $\mathcal{O}_{L}$ lying above $p$, and let $L_{\wp}$ be the completion of $L$ at $\wp$. Let $E$ be an elliptic curve defined over $L$ with potential good (ordinary or supersingular) reduction at $\wp$. Let us fix an embedding $\iota: \bar{L} \hookrightarrow \bar{L}_{\wp}$. Via $\iota$, we may regard $E$ as defined over $L_{\wp}$. Let $L_{\wp}^{\mathrm{nr}}$ be the maximal unramified extension of $L_{\wp}$.

We follow Serre and Tate (see in particular [11] p. 498, Cor. 3) to define an extension $K_{E}$ of $L_{\wp}^{\mathrm{nr}}$ of minimal degree such that $E$ has good reduction over $K_{E}$. Let $\ell$ be any prime 
such that $\ell \neq p$, and let $T_{\ell}(E)$ be the $\ell$-adic Tate module. Let $\rho_{E, \ell}: \operatorname{Gal}\left(\overline{L_{\wp}^{\mathrm{nr}}} / L_{\wp}^{\mathrm{nr}}\right) \rightarrow$ $\operatorname{Aut}\left(T_{\ell}(E)\right)$ be the usual representation induced by the action of Galois on $T_{\ell}(E)$. We define the field $K_{E}$ as the extension of $L_{\wp}^{\mathrm{nr}}$ such that

$$
\operatorname{Ker}\left(\rho_{E, \ell}\right)=\operatorname{Gal}\left(\overline{L_{\wp}^{\mathrm{nr}}} / K_{E}\right) .
$$

In particular, the field $K_{E}$ enjoys the following properties:

1. $E / K_{E}$ has good (ordinary or supersingular) reduction.

2. $\quad K_{E}$ is the smallest extension of $L_{\wp}^{\mathrm{nr}}$ such that $E / K_{E}$ has good reduction, i.e., if $K^{\prime} / L_{\wp}^{\mathrm{nr}}$ is another extension such that $E / K^{\prime}$ has good reduction, then $K_{E} \subseteq K^{\prime}$.

3. $K_{E} / L_{\wp}^{\mathrm{nr}}$ is finite and Galois. Moreover (see [10], $\S 5.6$, p. 312 when $L=\mathbb{Q}$, but the same reasoning holds over number fields, as the work of Néron is valid for any local field, [8] p. 124-125):

- If $p>3$, then $K_{E} / L_{\wp}^{\mathrm{nr}}$ is cyclic of degree $1,2,3,4$, or 6 .

- If $p=3$, the degree of $K_{E} / L_{\wp}^{\mathrm{nr}}$ is a divisor of 12 .

- If $p=2$, the degree of $K_{E} / L_{\wp}^{\mathrm{nr}}$ is $2,3,4,6,8$, or 24 .

Example 2.1. Let $E=E_{27 a 4}$ be the elliptic curve with Cremona label "27a4", with $j$ invariant $j(E)=-2^{15} \cdot 3 \cdot 5^{3}$, given by a Weierstrass equation

$$
y^{2}+y=x^{3}-30 x+63 .
$$

The elliptic curve $E$ has bad additive reduction at $p=3$. The extension $K=K_{E}$ of $\mathbb{Q}_{3}^{\mathrm{nr}}$ is given by adjoining $\alpha=\sqrt[4]{3}$ and a $\operatorname{root} \beta$ of $x^{3}-120 x+506=0$. The result is an extension $K=\mathbb{Q}_{3}^{\mathrm{nr}}(\alpha, \beta)$ of degree $e=12$.

Let $(\pi)$ be the unique prime ideal of $K$ above (3). Let $E^{\prime} / K$ be an elliptic curve isomorphic to $E$ over $K$ given by an integral model, minimal at $(\pi)$, with good reduction at $\wp$. The reduction of $E^{\prime} / A$ modulo $\pi$ is given by $y^{2} \equiv x^{3}+x+2$ over $\mathbb{F}_{3}$, which is a supersingular elliptic curve. Thus, $E / K$ is an elliptic curve with supersingular good reduction at the prime above $p=3$.

Example 2.2. Let $E=E_{121 \mathrm{c} 2}$ be the elliptic curve with Cremona label "121c2", with $j$-invariant $j(E)=-11 \cdot 131^{3}$, and discriminant $\Delta=-11^{8}$, given by a Weierstrass equation

$$
y^{2}+x y=x^{3}+x^{2}-3632 x+82757 .
$$

The elliptic curve $E$ has bad additive reduction at $p=11$, but potential good supersingular reduction at the same prime. The extension $K=K_{E}$ of $\mathbb{Q}_{11}^{\mathrm{nr}}$ is given by adjoining $\pi=\sqrt[3]{11}$, thus $e=3$. The curve $E$ has a minimal model with good supersingular reduction of the form

$$
y^{2}+\sqrt[3]{11} x y=x^{3}+\sqrt[3]{11^{2}} x^{2}+3 \sqrt[3]{11} x+2
$$

over $\mathbb{Q}_{11}^{\mathrm{nr}}(\pi)$, where $\pi=\sqrt[3]{11}$, and the discriminant of this model is $\Delta=-1$.

Let $e$ be the ramification index of $K / \mathbb{Q}_{p}$. Since $e / e(\wp \mid p)=\left[K_{E}: L_{\wp}^{\mathrm{nr}}\right]$, the value of $e$ can be obtained directly from $e(\wp \mid p)$ and a model of $E / L$, thanks to the classification of Néron models. As a reference for the following theorem, the reader can consult [8], p. 124-125, or [10], §5.6, p. 312, where $\operatorname{Gal}\left(K_{E} / L_{\wp}^{\mathrm{nr}}\right)$ is denoted by $\Phi_{p}$, and therefore $e / e(\wp \mid p)=$ 
$\operatorname{Card}\left(\Phi_{p}\right)$. Notice, however, that the section we cite of [10] restricts its attention to the case $L=\mathbb{Q}$.

Theorem 2.3. Let $p>3$, let $E / L$ be an elliptic curve with potential good reduction, and let $\Delta_{L}$ be the discriminant of any model of $E$ defined over $L$. Let $K_{E}$ be the smallest extension of $L_{\wp}^{n r}$ such that $E / K_{E}$ has good reduction. Then e/e $(\wp \mid p)=\left[K_{E}: L_{\wp}^{n r}\right]=1,2,3,4$, or 6 . Moreover:

- $e / e(\wp \mid p)=2$ if and only if $v_{\wp}\left(\Delta_{L}\right) \equiv 6 \bmod 12$,

- $e / e(\wp \mid p)=3$ if and only if $v_{\wp}\left(\Delta_{L}\right) \equiv 4$ or $8 \bmod 12$,

- $e / e(\wp \mid p)=4$ if and only if $v_{\wp}\left(\Delta_{L}\right) \equiv 3$ or $9 \bmod 12$,

- $e / e(\wp \mid p)=6$ if and only if $v_{\wp}\left(\Delta_{L}\right) \equiv 2$ or $10 \bmod 12$.

Example 2.4. Let $E=E_{121 \mathrm{c} 2}$, defined over $L=\mathbb{Q}$, so $e(\wp \mid p)=1$. Since $v_{\wp}(\Delta)=8$, we conclude that $e=3$ by Theorem 2.3 , which agrees with $K_{E}=\mathbb{Q}_{11}^{\mathrm{nr}}(\sqrt[3]{11})$ as we saw in Example 2.2.

Let $K=K_{E}$, and let $v_{K}$ be a valuation on $K$ such that $v_{K}(p)=e$ and $\nu_{K}(\pi)=1$, where $\pi$ is a uniformizer for $K$. Let $A$ be the ring of elements of $K$ with valuation $\geq 0$, let $\mathcal{M}$ be the maximal ideal of $A$, and let $\mathbb{F}=A / \mathcal{M}$ be the residue field of $K$. We fix a minimal model of $E$ over $A$ with good reduction, given by

$$
y^{2}+a_{1} x y+a_{3} y=x^{3}+a_{2} x^{2}+a_{4} x+a_{6},
$$

with $a_{i} \in A$. In particular, the discriminant $\Delta$ is a unit in $A$. Moreover, since $E / A$ has good reduction, we have an exact sequence

$$
0 \rightarrow X_{p^{n}} \rightarrow E(\bar{K})\left[p^{n}\right] \rightarrow \widetilde{E}(\overline{\mathbb{F}})\left[p^{n}\right] \rightarrow 0,
$$

where $\pi_{n}: E(\bar{K})\left[p^{n}\right] \rightarrow \widetilde{E}(\overline{\mathbb{F}})\left[p^{n}\right]$ is the homomorphism given by reduction modulo $\mathcal{M}$, and $X_{p^{n}}$ is the kernel of $\pi_{n}$ (see [12], Ch. VII, Thm. 2.1). By taking inverse limits and tensoring with $\mathbb{Q}_{p}$, we obtain another exact sequence

$$
0 \rightarrow X \rightarrow V_{p}(E) \rightarrow V_{p}(\widetilde{E}) \rightarrow 0,
$$

where $X=\left(\lim _{\leftarrow} X_{p^{n}}\right) \otimes \mathbb{Q}_{p}$, and $V_{p}(E)=T_{p}(E) \otimes \mathbb{Q}_{p}$. We distinguish two cases, according to whether the Hasse invariant of $E / \mathbb{F}$ is non-zero (ordinary reduction) or zero (supersingular reduction).

In this paper, we only discuss the supersingular reduction case (the multiplicative and the ordinary case will be treated in [6]). We assume from now on that $E$ is an elliptic curve defined over $L$ with potential good supersingular reduction at $\wp$. Let $\iota, K=K_{E}$, and $A$ be as before. We fix a minimal model of $E$ over $A$ with good reduction, given by

$$
y^{2}+a_{1} x y+a_{3} y=x^{3}+a_{2} x^{2}+a_{4} x+a_{6},
$$

with $a_{i} \in A$. Let $\widehat{E} / A$ be the formal group associated to $E / A$, with formal group law given by a power series $F(X, Y) \in A[[X, Y]]$, as defined in Ch. IV of [12]. Let

$$
[p](Z)=\sum_{i=1}^{\infty} s_{i} Z^{i}
$$


be the multiplication-by- $p$ homomorphism in $\widehat{E}$, for some $s_{i} \in A$ for all $i \geq 1$. Since $E / K$ has good supersingular reduction, the formal group $\widehat{E} / A$ associated to $E$ has height 2 (see [12], Ch. V, Thm. 3.1). Thus, $s_{1}=p$ and the coefficients $s_{i}$ satisfy $v_{K}\left(s_{i}\right) \geq 1$ if $i<p^{2}$ and $v_{K}\left(s_{p^{2}}\right)=0$. Let $q_{0}=1, q_{1}=p$ and $q_{2}=p^{2}$, and put $e_{i}=v_{K}\left(s_{q_{i}}\right)$. In particular $e_{0}=v_{K}\left(s_{1}\right)=v_{K}(p)=e$, and $e_{1}=v_{K}\left(s_{p}\right)$, and $e_{2}=v_{K}\left(s_{p^{2}}\right)=0$. Then, the multiplication-by- $p$ map can be expressed as

$$
[p](Z)=p f(Z)+\pi^{e_{1}} g\left(Z^{p}\right)+h\left(Z^{p^{2}}\right),
$$

where $f(Z), g(Z)$ and $h(Z)$ are power series in $Z \cdot A[[Z]]$, with $f^{\prime}(0)=g^{\prime}(0)=h^{\prime}(0) \in A^{\times}$. The value of $e_{1}$ is independent of the chosen minimal model for $E / A$ (see [4], Cor. 3.2).

Example 2.5. Let $E / \mathbb{Q}$ be the elliptic curve with Cremona label "27a4" as in Example 2.1. The multiplication-by-3 map on the associated formal group $\widehat{E}$ is given by a power series:

$$
[3](Z)=3 Z+s_{3} Z^{3}+O\left(Z^{4}\right),
$$

where $v_{K}\left(s_{3}\right)=2$. Hence, $e_{1}=2$ in this case. (The number $s_{3}$ was given in Example 2.2 of [4]. We will calculate $e_{1}$ in a different way below, in Example 3.4.)

Example 2.6. Let $E=E_{121 \mathrm{c} 2}$ be the elliptic curve with Cremona label "121c2". The multiplication-by-11 map on the associated formal group $\widehat{E}$ is given by a power series:

$$
\begin{aligned}
{[11](Z)=} & 11 Z-55 \pi Z^{2}-275 \pi^{2} Z^{3}+42350 Z^{4}-181148 \pi Z^{5}-659417 \pi^{2} Z^{6} \\
& +96265708 Z^{7}-341161040 \pi Z^{8}-1521191342 \pi^{2} Z^{9}+183261837077 Z^{10} \\
& -497606935519 \pi Z^{11}+O\left(Z^{12}\right) .
\end{aligned}
$$

Since $497606935519=17 \cdot 23 \cdot 151 \cdot 8428159$ is relatively prime to 11 , we conclude that

$$
e_{1}=v_{K}\left(s_{11}\right)=v_{K}(-497606935519 \pi)=1 \text {. }
$$

\section{Previous results}

In [4], the author has shown several results on the values of $e$ and $e_{1}$, that we quote here for the convenience of the reader. Before we state the results, we define quantities $r(p)$ and $s(p)$ for each prime $p>3$, by

$$
r(p)=\left\{\begin{array}{l}
1, \text { if } p \equiv 5 \text { or } 11 \bmod 12, \\
0, \text { if } p \equiv 1 \text { or } 7 \bmod 12,
\end{array} \text { and } s(p)=\left\{\begin{array}{l}
1, \text { if } p \equiv 3 \bmod 4, \\
0, \text { if } p \equiv 1 \bmod 4 .
\end{array}\right.\right.
$$

Equivalently, $r(p)=\frac{1}{2}\left(1-\left(\frac{-3}{p}\right)\right)$ and $s(p)=\frac{1}{2}\left(1-\left(\frac{-4}{p}\right)\right)$, where $\left(\frac{\dot{p}}{p}\right)$ is the Legendre symbol. We also need to define a polynomial $Q_{p}(T)$, whose existence and properties were shown in Lemma 3.6 of [4].

Definition 3.1. Let $p>3$ be a prime and let $P_{p}(X, Y)$ be the polynomial in $\mathbb{Z}[X, Y]$ defined by

$$
P_{p}(X, Y)=\sum_{\substack{m, n \geq 0 \\
4 m+6 n=p-1}}(-1)^{m+n}\left(\begin{array}{c}
\frac{p-1}{2} \\
m+n
\end{array}\right)\left(\begin{array}{c}
m+n \\
m
\end{array}\right)(27 X)^{m}(54 Y)^{n} .
$$


We define $Q_{p}(T) \in \mathbb{Z}[T]$ as the unique polynomial with integer coefficients such that

$$
P_{p}(X, Y)=X^{r(p)} Y^{s(p)} \Delta^{\left\lfloor\frac{p}{12}\right\rfloor} Q_{p}(j)
$$

where $\Delta$ and $j$ are defined by $1728 \Delta=X^{3}-Y^{2}$ and $\Delta \cdot j=X^{3}$, where $\lfloor\cdot\rfloor$ is the greatest integer function.

Example 3.2. For instance,

$$
P_{5}=-54 X, P_{7}=-162 Y, P_{11}=29160 X Y \text {, }
$$

and

$$
P_{13}=-393660 X^{3}+43740 Y^{2}=\Delta(E)(-349920 j(E)-75582720) .
$$

The corresponding polynomials $Q_{p}(T)$ are:

$$
Q_{5}(T)=-54, Q_{7}(T)=-162, Q_{11}(T)=29160, Q_{13}(T)=-349920 T-75582720 \text {. }
$$

Theorem 3.3 ([4], Thm. 3.9). Let E/L be an elliptic curve with potential good supersingular reduction at a prime $\wp$ above a prime $p$. Let $K=K_{E}$ be the extension of $L_{\wp}^{n r}$ defined above, let $A, e=v_{K}(p)$, and $e_{1}$ be as before, and let $e(\wp \mid p)$ be the ramification index of $\wp$ in $L / \mathbb{Q}$. Let $y^{2}+a_{1} x y+a_{3} y=x^{3}+a_{2} x^{2}+a_{4} x+a_{6}$ be a minimal model for $E / A$ with good reduction, and let $c_{4}, c_{6} \in A$ be the usual quantities associated to this model (as defined in [12], Chapter III, § 1). Then:

1. If $p=2$, and $\frac{v_{K}\left(c_{4}\right)}{4}<e$, then

$$
e_{1}=\frac{v_{K}\left(c_{4}\right)}{4}=\frac{v_{K}(j(E))}{12}=\frac{e \cdot v_{\wp}(j(E))}{12 e(\wp \mid p)} .
$$

2. If $p=3$, and $\frac{v_{K}\left(c_{4}\right)}{2}<e$, then

$$
e_{1}=\frac{v_{K}\left(c_{4}\right)}{2}=\frac{v_{K}(j(E))}{6}=\frac{e \cdot v_{\wp}(j(E))}{6 e(\wp \mid p)} .
$$

3. If $p>3$, and $\lambda=r(p) v_{K}\left(c_{4}\right)+s(p) v_{K}\left(c_{6}\right)+v_{K}\left(Q_{p}(j(E))\right)<e$, then

$$
\begin{aligned}
e_{1} & =\lambda \\
& =r(p) \frac{v_{K}(j(E))}{3}+s(p) \frac{v_{K}(j(E)-1728)}{2}+v_{K}\left(Q_{p}(j(E))\right) \\
& =\frac{e}{e(\wp \mid p)} \cdot\left(r(p) \frac{v_{\wp}(j(E))}{3}+s(p) \frac{v_{\wp}(j(E)-1728)}{2}+v_{\wp}\left(Q_{p}(j(E))\right)\right) .
\end{aligned}
$$

Otherwise, $e_{1} \geq e$.

Example 3.4. In Example 2.5 we looked at the elliptic curve $E / \mathbb{Q}$ with label "27a4", for $p=3$, and concluded that $e_{1}=2$. Alternatively, and much easier to compute, we use Theorem 3.3:

$$
\lambda=\frac{e \cdot v_{3}(j(E))}{6}=\frac{\left.12 \cdot v_{3}\left(-2^{15} \cdot 3 \cdot 5^{3}\right)\right)}{6}=2 .
$$

Since $2=\lambda<e=12$, we conclude that $e_{1}=\lambda=2$.

If we combine Theorems 3.3 and 2.3, then we reach the following corollary. 
Corollary 3.5. Let $p>3$ be a prime and let $E / L$ be an elliptic curve with potential supersingular good reduction at a prime $\wp$ above $p$. Let e $(\wp \mid p)$ be the ramification index of $\wp$ in $L / \mathbb{Q}$. Let $j(E) \in L$ be its $j$-invariant, let $\Delta_{L}$ be the discriminant of a model for $E$ over $L$, and define an integer $\lambda$ as follows:

- If $v_{\wp}\left(\Delta_{L}\right) \equiv 6 \bmod 12$, then $e / e(\wp \mid p)=2$. Let

$$
\lambda=\frac{2}{3} r(p) v_{\wp}(j(E))+s(p) v_{\wp}(j(E)-1728)+2 v_{\wp}\left(Q_{p}(j(E))\right),
$$

- If $v_{\wp}\left(\Delta_{L}\right) \equiv 4$ or $8 \bmod 12$, then $e / e(\wp \mid p)=3$. Let

$$
\lambda=r(p) v_{\wp}(j(E))+\frac{3}{2} s(p) v_{\wp}(j(E)-1728)+3 v_{\wp}\left(Q_{p}(j(E))\right),
$$

- If $v_{\wp}\left(\Delta_{L}\right) \equiv 3$ or $9 \bmod 12$, then $e / e(\wp \mid p)=4$. Let

$$
\lambda=\frac{4}{3} r(p) v_{\wp}(j(E))+2 s(p) v_{\wp}(j(E)-1728)+4 v_{\wp}\left(Q_{p}(j(E))\right),
$$

- If $v_{\wp}\left(\Delta_{L}\right) \equiv 2$ or $10 \bmod 12$, then $e / e(\wp \mid p)=6$. Let

$$
\lambda=2 r(p) v_{\wp}(j(E))+3 s(p) v_{\wp}(j(E)-1728)+6 v_{\wp}\left(Q_{p}(j(E))\right) .
$$

If $\lambda<e$, then $e_{1}=\lambda$. Otherwise, if $\lambda \geq e$, then $e_{1} \geq e$.

Example 3.6. Let us return to the curve $E / \mathbb{Q}$ with label "121c2". In Examples 2.2 and 2.4 we showed a minimal model over $\mathbb{Q}_{11}^{\text {nr }}(\sqrt[3]{11})$ and we proved that $e_{1}=1$. We may also verify this value using the formula in Corollary 3.5. Here $p=11$, so $r(11)=s(11)=1$, and $L=\mathbb{Q}$, so $e(\wp \mid p)=1$. The discriminant of the model for $E / \mathbb{Q}$ given in Example 2.2 is $\Delta_{\mathbb{Q}}=-11^{8}$, we have $j(E)=-11 \cdot 131^{3}$ and $j(E)-1728=-4973^{2}$. Hence:

$$
\lambda=r(p) v_{p}(j(E))+\frac{3}{2} s(p) v_{p}(j(E)-1728)+3 v_{p}\left(Q_{p}(j(E))\right)=1 \cdot 1+\frac{3}{2} \cdot 1 \cdot 0+3 \cdot 0=1 .
$$

and so, $e_{1}=\lambda=1$.

\section{Additional results on the formal group}

As we will show below in Corollary 4.2, the values of $e$ and $e_{1}$ are restricted to certain values in certain cases. First, we need a lemma.

Lemma 4.1. Let $p>3$ be a prime.

1. If $v_{\wp}(j)>0$, then $v_{\wp}\left(Q_{p}(j)\right)=0$.

2. If $v_{\wp}(j-1728)>0$ and $v_{\wp}(j)=0$, then $v_{\wp}\left(Q_{p}(j)\right)=0$.

Proof. It follows from the formulae in [4], Lemma 3.6 and Remark 3.8, that

$$
Q_{p}(T)=q_{d} T^{d}+\ldots+q_{1} T+q_{0}=\sum d_{f} T^{f}(T-1728)^{\left\lfloor\frac{p}{12}\right\rfloor-f},
$$

where $f, g \geq 0$, and $0 \leq f \leq\left\lfloor\frac{p}{12}\right\rfloor$, and

$$
d_{f}=(-1)^{m+n} \cdot\left(\begin{array}{c}
\frac{p-1}{2} \\
m+n
\end{array}\right) \cdot\left(\begin{array}{c}
m+n \\
m
\end{array}\right) \cdot 27^{m} \cdot 54^{n},
$$

where $m=3 f+r(p)$, and $n=2 g+s(p)$. It follows that the constant term in $Q_{p}(T)$ is given by $q_{0}=d_{0} \cdot(-1728)^{\left\lfloor\frac{p}{12}\right\rfloor}$. When $f=0$ we have $m=r(p)$ and $n=2 \cdot\left\lfloor\frac{p}{12}\right\rfloor+s(p)$. 
Then the constant term of $Q_{p}(T)$ is given by:

$$
q_{0}=d_{0} \cdot(-1728)^{\left\lfloor\frac{p}{12}\right\rfloor}=(-1)^{m+n} \cdot\left(\begin{array}{c}
\frac{p-1}{2} \\
m+n
\end{array}\right) \cdot\left(\begin{array}{c}
m+n \\
m
\end{array}\right) \cdot 27^{m} \cdot 54^{n} \cdot(-1728)^{\left\lfloor\frac{p}{12}\right\rfloor} .
$$

Since $p>3$, the constant term $q_{0}$ is not divisible by $p$. If $v_{\wp}(j)>0$, then $v_{\wp}\left(Q_{p}(j)\right)=$ $v_{\wp}\left(q_{0}\right)=0$. This shows part (1).

For part (2), note that we may write

$$
Q_{p}(j)=d_{\left\lfloor\frac{p}{\lfloor 2}\right\rfloor} j^{\left\lfloor\frac{p}{12}\right\rfloor}+\sum_{0 \leq f<\left\lfloor\frac{p}{12}\right\rfloor} d_{f} j^{f}(j-1728)^{\left\lfloor\frac{p}{12}\right\rfloor-f} .
$$

If we set $m=3 \cdot\left\lfloor\frac{p}{12}\right\rfloor+r(p)$ and $n=s(p)$, then the coefficient $d_{\left\lfloor\frac{p}{12}\right\rfloor}$ is given by

$$
d_{\left\lfloor\frac{p}{12}\right\rfloor}=(-1)^{m+n} \cdot\left(\begin{array}{c}
\frac{p-1}{2} \\
m+n
\end{array}\right) \cdot\left(\begin{array}{c}
m+n \\
m
\end{array}\right) \cdot 27^{m} \cdot 54^{n} .
$$

Since $d_{\left\lfloor\frac{p}{12}\right\rfloor}$ is not divisible by $p>3$, and $v_{\wp}(j)=0$ and $v_{\wp}(j-1728)>0$ by assumption, it follows that $v_{\wp}\left(Q_{p}(j)\right)=0$, as desired.

The following result extends Corollary 4.6 of [4], which only covered the case when $e(\wp \mid p)=1$.

Corollary 4.2. Let $E / L$ be an elliptic curve with potential supersingular reduction at a prime $\wp$ lying above a prime $p>3$, and let $e$ and $e_{1}$ be defined as in Section 2. Assume that $e_{1}<e$. Then,

1. If $j(E) \not \equiv 0$ or $1728 \bmod \wp$, then $e_{1}=e / e(\wp \mid p) \cdot v_{\wp}\left(Q_{p}(j)\right)$;

2. If $j(E) \equiv 0 \bmod \wp$, then $e_{1}=e \cdot v_{\wp}(j) / 3 e(\wp \mid p)$, with $1 \leq v_{\wp}(j)<3 e(\wp \mid p)$. If $v_{\wp}(j)$ is not divisible by 3 , then $e / e(\wp \mid p)=3$ or 6 , and $e_{1}=v_{\wp}(j)$ or $2 v_{\wp}(j)$.

3. If $j(E) \equiv 1728 \bmod \wp$, then $e_{1}=e \cdot v_{\wp}(j-1728) / 2 e(\wp \mid p)$, with $1 \leq v_{\wp}(j-1728)<2 e(\wp \mid p)$. If $v_{\wp}(j-1728)$ is even, then $e / e(\wp \mid p)=2$ or 4 , and $e_{1}=v_{\wp}(j-1728)$ or $2 v_{\wp}(j-1728)$.

Proof. Let $p>3$ be a prime, assume that $e_{1}<e$, let $K_{E}$ be the extension of degree $e$ of $L_{\wp}^{\mathrm{nr}}$ defined above, and fix a minimal model of $E$ over $K_{E}$ with good supersingular reduction. Let $\Delta$ be its discriminant, and let $c_{4}$ and $c_{6}$ be the usual quantities. Let $\lambda=$ $r(p) v_{K}\left(c_{4}\right)+s(p) v_{K}\left(c_{6}\right)+v_{K}\left(Q_{p}(j(E))\right)$ as in Theorem 3.3. If $\lambda \geq e$ then $e_{1} \geq e$, but we have assumed that $e_{1}<e$, and hence $e_{1}=\lambda$. Let us write $e^{\prime}=e / e(\wp \mid p)$. In this case, $v_{K}\left(Q_{p}(j(E))\right)=e^{\prime} \cdot v_{\wp}\left(Q_{p}(j(E))\right)$ is a multiple of $e^{\prime}$. Under our assumptions

$$
\begin{aligned}
e_{1} & =r(p) v_{K}\left(c_{4}\right)+s(p) v_{K}\left(c_{6}\right)+v_{K}\left(Q_{p}(j)\right) \\
& =e^{\prime} \cdot\left(r(p) v_{\wp}\left(c_{4}\right)+s(p) v_{\wp}\left(c_{6}\right)+v_{\wp}\left(Q_{p}(j)\right)\right) .
\end{aligned}
$$

Since $v_{K}(\Delta)=0$ and $p \neq 2,3$, the equality $1728 \Delta=c_{4}^{3}-c_{6}^{2}$ implies that $v_{K}\left(c_{4}\right)$ and $v_{K}\left(c_{6}\right)$ cannot be simultaneously positive. We note that $c_{4}^{3} / \Delta=j$ and $c_{6}^{2}=\Delta \cdot(j-1728)$. Since $v_{K}(\Delta)=0$, it follows that $v_{K}\left(c_{4}\right)=v_{K}(j) / 3$ and $v_{K}\left(c_{6}\right)=v_{K}(j-1728) / 2$. Since $c_{4}, c_{6} \in L$, it follows that $v_{K}\left(c_{4}\right)=0$ (resp. $v_{K}\left(c_{6}\right)=0$ ) if and only if $v_{\wp}(j)=0$ (resp. $\left.v_{\wp}\left(c_{6}\right)=0\right)$, if and only if $j \neq \equiv 0 \bmod \wp($ resp. $j-1728 \not \equiv 0 \bmod \wp)$.

- If $v_{K}\left(c_{4}\right)=v_{K}\left(c_{6}\right)=0$, then $e_{1}=e^{\prime} \cdot v_{\wp}\left(Q_{p}(j)\right)$. 
- If $v_{K}\left(c_{4}\right)>0$ and $v_{K}\left(c_{6}\right)=0$, then $v_{K}(j(E))=v_{K}\left(c_{4}^{3} / \Delta\right)=3 v_{K}\left(c_{4}\right)>0$. Since $j(E) \in L$, it follows that $j(E) \equiv 0 \bmod \wp$. In particular, $v_{K}(j)=e^{\prime} \cdot v_{\wp}(j)$ is a multiple of $e / e(\wp \mid p)=e^{\prime}$, say $v_{K}(j)=e^{\prime} \cdot v_{\wp}(j)$. Theorem 3.3 and Corollary 4.1 say that

$$
e_{1}=r(p) v_{K}\left(c_{4}\right)+s(p) v_{K}\left(c_{6}\right)+v_{K}\left(Q_{p}(j)\right)=e^{\prime} \cdot\left(r(p) \frac{v_{\wp}(j)}{3}\right) \text {. }
$$

Thus, we must have $r(p)=1$ (in particular, $p \equiv 5 \bmod 6$ in this case) and $e_{1}=v_{K}\left(c_{4}\right)$, otherwise $0=e_{1} \geq 1$, a contradiction. Hence,

$$
e_{1}=v_{K}\left(c_{4}\right)=\frac{v_{K}(j)}{3}=\frac{e^{\prime} \cdot v_{\wp}(j)}{3} \text {. }
$$

Since $e_{1}<e$ by assumption, it follows that $1 \leq v_{\wp}(j)<3 e(\wp \mid p)$. In addition, $e_{1}$ is a positive integer, so $e^{\prime} v_{\wp}(j) \equiv 0 \bmod 3$. If $v_{\wp}(j)$ is not a multiple of 3 , then $e^{\prime} \equiv 0 \bmod 3$. Finally, $e^{\prime}=1,2,3,4$, or 6 , so $e^{\prime}=3$ or 6 in this case, and $e_{1}=v_{\wp}(j)$ or $2 v_{\wp}(j)$, as claimed.

- If instead we have $v_{K}\left(c_{4}\right)=0$ and $v_{K}\left(c_{6}\right)>0$, Theorem 3.3 and Corollary 4.1 now say that $e_{1}=v_{K}\left(c_{6}\right)$ (we must have $p \equiv 3 \bmod 4$ in this case). It follows that $j \equiv 1728 \bmod \wp$ and $v_{K}(j-1728)=e^{\prime} h$ where $h=v_{\wp}(j-1728) \geq 1$. Since $e_{1}<e$, we have $h<2 e(\wp \mid p)$. Since $e_{1}$ is an integer, and if $h$ is odd, then $e^{\prime} \equiv 0 \bmod 2$. Thus, $e^{\prime}=2$, 4, or 6 , and therefore, $e_{1}=h, 2 h$, or $3 h$. However, we shall show next that $j \equiv 1728 \bmod \wp$ and $e^{\prime}=6$ is not possible. Thus, $e_{1}=h$, or $2 h$, and the proof of the corollary would be finished.

Indeed, suppose $j \equiv 1728 \bmod \wp$ and $e^{\prime}=6$. Let $\Delta_{L}, c_{4, L}$ and $c_{6, L}$ be the discriminant and the usual constants associated to the original model of $E$ over $L$. By the work of Néron on minimal models (Theorem 2.3), the degree $e^{\prime}=6$ occurs if and only if $v_{\wp}\left(\Delta_{L}\right) \equiv 2$ or $10 \bmod 12$. Since $\Delta_{L} \cdot j(E)=\left(c_{4, L}\right)^{3}$, and $j \equiv 1728 \bmod \wp$, with $p>3$, it follows that

$$
v_{\wp}\left(\Delta_{L}\right)=3 v_{\wp}\left(c_{4, L}\right)
$$

and therefore $v_{\wp}\left(\Delta_{L}\right) \equiv 0 \bmod 3$, and we cannot have $v_{\wp}\left(\Delta_{L}\right) \equiv 2$ or $10 \bmod 12$. This is a contradiction, and therefore $e^{\prime}=6$ and $j \equiv 1728 \bmod \wp$ are incompatible. This ends the proof of the corollary.

Corollary 4.3. With notation as in Corollary 4.2, if $e_{1}<e$, and $p>\max \{3 e(\wp \mid p)-1,3\}$, then $p e /(p+1)>e_{1}$.

Proof. Suppose $e_{1}<e$. According to Corollary 4.2, the biggest possible value of $e_{1}$ is $(3 e(\wp \mid p)-1) e / 3 e(\wp \mid p)$. Since the function $k(x-1) / x$ is increasing for any $k>0$ and any $x>1$, it follows that if $p+1>3 e(\wp \mid p)$, then

$$
e_{1} \leq \frac{(3 e(\wp \mid p)-1) e}{3 e(\wp \mid p)}<\frac{p e}{p+1} .
$$

Example 4.4. Here we illustrate the last three results with the curve $E / \mathbb{Q}$ with label "121c2". The discriminant of the chosen model for $E / \mathbb{Q}$ is $\Delta_{\mathbb{Q}}=-11^{8}$, we have $j(E)=-11 \cdot 131^{3}$ and $j(E)-1728=-4973^{2}$. 
Since $v_{11}(j)=1$, Lemma 4.1 implies that $v_{11}\left(Q_{11}(j)\right)=0$. Indeed, we know that $Q_{11}(j)$ is constant, equal to $29160=2^{3} \cdot 3^{6} \cdot 5$, so its 11 -adic valuation is zero.

Moreover, $v_{11}(j)=1>0$ so Corollary 4.2 says that $e_{1}=e \cdot v_{\wp}(j) / 3 e(\wp \mid p)=(3 \cdot 1) / 3=$ 1, as we had already computed in Example 2.6.

Finally, we have $p e /(p+1)=33 / 12>1=e_{1}$, in agreement with Corollary 4.3.

The next three results concern not only $e$ and $e_{1}$, but also the values of $e-p^{u} e_{1}$, for every $u \geq 0$ such that $e-p^{u} e_{1} \geq 1$. As we shall see later (Lemma 5.3 and Proposition 5.6), these values are closely related to the constants $c(E / L, R, \wp)$ of Theorem 1.2 in the introduction.

Corollary 4.5. Let $\eta \geq 1$ be a fixed positive integer. Let $L$ be a number field, and let $\wp$ be a prime ideal of $\mathcal{O}_{L}$ lying above a prime $p$, such that $e(\wp \mid p)=\eta$. Let $E / L$ be an elliptic curve with potential good supersingular reduction at $\wp$. Then, there is a constant $f(\eta, p)$, which depends only on $\eta$ when $p>3$ (and does not on $\wp, L$, or E), such that $e$ is a divisor of $f(\eta, p)$, and if $e_{1}<e$, then the quantities $e_{1}$, and $e-p^{u} e_{1}$, for every $u \geq 0$ such that $e-p^{u} e_{1} \geq 1$, are also divisors off $(\eta, p)$. Moreover,

1. For any $p$, the constant $f(\eta, p)$ is a divisor of $F(\eta)=\operatorname{lcm}(\{n: 1 \leq n<24 \eta$, $\operatorname{gcd}(n, 6) \neq 1\})$.

2. The constant $f(\eta, 2)$ is a divisor of $F(\eta, 2)=\operatorname{lcm}(\{2 n: 1 \leq n<12 \eta\})$.

3. The constant $f(\eta, 3)$ is a divisor of $F(\eta, 3)=\operatorname{lcm}(\{2 n: 1 \leq n<6 \eta\})$.

4. If $p>3$, the constant $f(\eta, p)$ is a divisor of $F_{0}(\eta)=\operatorname{lcm}(\{n: 1 \leq n<6 \eta$, $\operatorname{gcd}(n, 6) \neq 1\})$.

5. If $\eta=1$ and $p>3$, then e divides 4 or 6 , and $e_{1}$ and $e-e_{1}$ are divisors of 4 .

Proof. Let $\eta \geq 1, L, \wp, e(\wp \mid p)=\eta$, and $E / L$ be as in the statement. We shall write $e^{\prime}=$ $e / e(\wp \mid p)$. Notice that, as defined, the quantities $F_{0}(\eta), F(\eta, 2)$, and $F(\eta, 3)$, are divisors of $F(\eta)$, so to show (1) through (4) it suffices to show that $f(\eta, p)$ divides $F(\eta, p)$ for $p=2,3$, and $f(\eta, p)$ divides $F_{0}(\eta)$ for $p>3$.

By our discussion at the beginning of Section 2, we have $e=e^{\prime} \eta$, where $e^{\prime}$ is a divisor of 24,12 , or 6 according to whether $p=2,3$, or $>3$, respectively. Thus, $e$ is clearly a divisor of $F(\eta, p)$, for all $p=2$ or 3 , and a divisor of $F_{0}(\eta)$ for $p>3$.

Let us assume from now on that $e_{1}<e$. If $p=2$, then Theorem 3.3 says that $e_{1}=e^{\prime} \cdot t / 12$, and we must have $1 \leq t<12 \eta$ to satisfy $e_{1}<e$. Since $p=2$, the number $e^{\prime}$ is a divisor of 24. Hence, $e_{1}$ is a divisor of $2 t$, with $1 \leq t<12 \eta$, and $e-p^{u} e_{1}=e^{\prime}\left(\eta-p^{u} t / 12\right) \geq 1$ is a divisor of $2\left(12 \eta-p^{u} t\right)$. It follows that both $e_{1}$ and $e-p^{u} e_{1}$ are divisors of $F(\eta, 2)=\operatorname{lcm}(\{2 n: 1 \leq n<12 \eta\})$. The number $e$ is a divisor of $24 \eta$. Since $24 \eta=\operatorname{lcm}(8 \eta, 3 \eta)$, then $e$ divides $F(\eta, 2)$.

If $p=3$, then Theorem 3.3 says that $e_{1}=e^{\prime} \cdot t / 6$, and we must have $1 \leq t<12 \eta$. Since $p=3$, the number $e^{\prime}$ is a divisor of 12 . Hence, $e_{1}$ is a divisor of $2 t$, with $1 \leq t<12 \eta$, and $e-p^{u} e_{1}=e^{\prime}\left(\eta-p^{u} t / 6\right)$ is a divisor of $2\left(12 \eta-p^{u} t\right)$. It follows that both $e_{1}$ and $e-p^{u} e_{1}$ are divisors of $F(\eta, 3)=\operatorname{lcm}(\{2 n: 1 \leq n<12 \eta\})$. The number $e$ is a divisor of $12 \eta$. Since $12 \eta=\operatorname{lcm}(4 \eta, 3 \eta)$, then $e$ divides $F(\eta, 3)$.

Now assume that $p>3$. It follows that $e^{\prime}$ is $1,2,3,4$, or 6 . In particular, $e^{\prime}$ is a divisor of 4 or 6. By Corollary 4.2, we have $e_{1}=e^{\prime} \cdot r$ with $1 \leq r<\eta$, or $e_{1}=e^{\prime} \cdot s / 3$ with $e_{1} \in \mathbb{Z}$ and 
$1 \leq s<3 \eta$, or $e_{1}=e^{\prime} \cdot t / 2$ with $e_{1} \in \mathbb{Z}$ and $1 \leq t<2 \eta$. In particular, $e_{1}$ is a divisor of a number in the set

$$
\{4 \alpha: 1 \leq \alpha<\eta\} \cup\{2 \beta: 1 \leq \beta<3 \eta\} \cup\{2 \gamma: 1 \leq \gamma<2 \eta\} \cup\{3 \delta: 1 \leq \delta<2 \eta\}
$$

Note that $\{4 \alpha: 1 \leq \alpha<\eta\} \subseteq\{2 \beta: 1 \leq \beta<3 \eta\}$, and so the number $e_{1}$ is a divisor of a number in the set

$$
\{2 \beta: 1 \leq \beta<3 \eta\} \cup\{3 \delta: 1 \leq \delta<2 \eta\}=\{n: 1 \leq n<6 \eta, \operatorname{gcd}(n, 6) \neq 1\} .
$$

Similarly, $e-p^{u} e_{1}=e^{\prime}\left(\eta-p^{u} r\right)$ with $1 \leq p^{u} r<\eta$, or $e-p^{u} e_{1}=e^{\prime}\left(\eta-p^{u} s / 3\right)$ with $e_{1} \in \mathbb{Z}$ and $1 \leq p^{u} s<3 \eta$, or $e_{1}=e^{\prime}\left(\eta-p^{u} t / 2\right)$ with $e_{1} \in \mathbb{Z}$ and $1 \leq p^{u} t<2 \eta$. Hence, $e-p^{u} e_{1}$ is a divisor of a number in the set

$\{4(\eta-\lambda): 1 \leq \lambda<\eta\} \cup\{2(3 \eta-\mu): 1 \leq \mu<3 \eta\} \cup\{2(2 \eta-\psi): 1 \leq \psi<2 \eta\} \cup$

$\{3(2 \eta-\rho): 1 \leq \rho<2 \eta\}=\{4 \alpha: \alpha<\eta\} \cup\{2 \beta: \beta<3 \eta\} \cup\{2 \gamma: \gamma<2 \eta\} \cup\{3 \delta: \delta<2 \eta\}$.

Therefore, $e-p^{u} e_{1}$ also is a divisor of a number in $\{n: 1 \leq n<6 \eta, \operatorname{gcd}(n, 6) \neq 1\}$. Hence, both $e_{1}$ and $e-p^{u} e_{1}$ are divisors of $\operatorname{lcm}(\{n: 1 \leq n<6 \eta, \operatorname{gcd}(n, 6) \neq 1\})$. The number $e$ is a divisor of $4 \eta$ or $6 \eta$. Since $4 \eta$ divides $F_{0}(\eta)$ and $6 \eta=\operatorname{lcm}(2 \eta, 3 \eta)$, then $e$ divides $F_{0}(\eta)$.

If $\eta=1$ and $1 \leq e_{1}<e$, then $e_{1}=e^{\prime} \cdot v_{\wp}\left(Q_{p}(j)\right)$ is impossible. Thus, by Corollary 4.2, either

- $e_{1}=e \cdot v_{\wp}(j) / 3$ with $1 \leq v_{\wp}(j)<3$. In this case $v_{\wp}(j)=1$ or 2 , so $e=3$ or 6 , and therefore $e_{1}=1,2$, or 4 . Note that $e_{1}=4$ can only happen if $e=6$, and if $e=6$, then $e_{1}=2$ or 4 . Since $p \geq 5$, $p e_{1}>e$, and we only need to consider $e, e_{1}$, and $e-e_{1}$. In particular, $e-e_{1}=1,2$, or 4 ; or

- $e_{1}=e \cdot v_{\wp}(j-1728) / 2$ with $1 \leq v_{\wp}(j-1728)<2$. In this case $v_{\wp}(j-1728)=1$, so $e=2$ or 4 , and therefore $e_{1}=1$, or 2 , respectively. Since $p \geq 5, p e_{1}>e$, and we only need to consider $e, e_{1}$, and $e-e_{1}$. Thus, $e-e_{1}=1$, or 2 , respectively.

Hence, in all cases $e$ divides 4 or 6 , and $e_{1}$ and $e-e_{1}$ are divisors of 4 .

Example 4.6. In previous examples we calculated $e=12$ and $e_{1}=2$ for the elliptic curve $E / \mathbb{Q}$ with label " $27 \mathrm{a} 4$ ”. Let us calculate $F(1,3)$. By definition

$$
F(1,3)=\operatorname{lcm}(\{2 n: 1 \leq n<6\})=\operatorname{lcm}(2,4,6,8,10)=8 \cdot 3 \cdot 5=120 .
$$

Thus, $e=12, e_{1}=2$, and $e-e_{1}=10$, and $e-3 e_{1}=6$ are divisors of $F(1,3)=120$, as predicted by Corollary 4.5 .

Example 4.7. Let $\eta \geq 1$, and put $F_{0}(\eta)=\operatorname{lcm}(\{n: 1 \leq n<6 \eta, \operatorname{gcd}(n, 6) \neq 1\})$. In this example we list a few values of $F_{0}(\eta)$ :

\begin{tabular}{l|l|l|l|l|l}
\hline$F_{0}(1)$ & $F_{0}(2)$ & $F_{0}(3)$ & $F_{0}(4)$ & $F_{0}(5)$ & $F_{0}(6)$ \\
\hline $2^{2} \cdot 3$ & $2^{3} \cdot 3^{2} \cdot 5$ & $2^{4} \cdot 3^{2} \cdot 5 \cdot 7$ & $2^{4} \cdot 3^{2} \cdot 5 \cdot 7 \cdot 11$ & $2^{4} \cdot 3^{3} \cdot 5 \cdot 7 \cdot 11 \cdot 13$ & $2^{5} \cdot 3^{3} \cdot 5 \cdot 7 \cdot 11 \cdot 13 \cdot 17$ \\
\hline
\end{tabular}

Corollary 4.8. With notation as in Corollary 4.2, if $p>3 e(\wp \mid p)$, then $e, e_{1}$, and $e-p^{u} e_{1} \geq 1$ are not divisible by $p$. 
Proof. Since $p>3$, Theorem 2.3 says that $e^{\prime}=e / e(\wp \mid p)$ is a divisor of 12 . Hence, if $p>3 e(\wp \mid p)$, then $e=e^{\prime} \cdot e(\wp \mid p)$ is relatively prime to $p$. By Corollary 4.5 , there is a constant $f(\eta)$ with $\eta=e(\wp \mid p)$ such that $e, e_{1}$ and $e-p^{u} e_{1}$ are divisors of $f(\eta)$, for all $u \geq 0$ such that $1 \leq e-p^{u} e_{1}$. Moreover, $f(\eta)$ can be chosen to be

$F(\eta)=\operatorname{lcm}(\{n: 1 \leq n<6 \eta, \operatorname{gcd}(n, 6) \neq 1\})=\operatorname{lcm}(\{2 \beta: 1 \leq \beta<3 \eta\} \cup\{3 \delta: 1 \leq \delta<2 \eta\})$.

Since $p>3 \eta$, there is no $\beta<3 \eta$ or $\delta<2 \eta$ such that $p$ is a divisor of $2 \beta$ or $3 \delta$. Thus, $f(\eta)$ is not divisible by $p$, and it follows that neither $e, e_{1}$ nor $e-p^{u} e_{1}$ is divisible by $p$.

Example 4.9. Let $E$ be the curve with label "121c2". We have previously calculated $e=3$ and $e_{1}=1$. The values $e=3, e_{1}=1$ and $e-e_{1}=2$ are divisors of $F_{0}(1)=12$, as stated in Corollary 4.5, and none of them are divisible by 11, as it follows from Corollary 4.8 .

We finish this section with two lemmas about quadratic twists of elliptic curves that we will need later on.

Lemma 4.10. Let $E / L$ be an elliptic curve with potential good reduction at a prime $\wp$ lying above a prime $p \geq 3$, and let $E^{\prime} / L$ be a quadratic twist of $E$. Let $F / L$ be the quadratic extension such that $E \cong_{F} E^{\prime}$. Let $K=K_{E}$ (resp. $K^{\prime}=K_{E^{\prime}}$ ) be the smallest extension of $L_{\wp}^{n r}$ such that $E / K$ (resp. $\left.E^{\prime} / K^{\prime}\right)$ has good reduction. Let $\pi$ and $\pi^{\prime}$ be uniformizers for $K$ and $K^{\prime}$ respectively, and let $v=v_{K}$ and $v^{\prime}=v_{K^{\prime}}$ be normalized valuations such that $v(\pi)=v^{\prime}\left(\pi^{\prime}\right)=1$. Let $e=v(p), e^{\prime}=v^{\prime}(p)$ and suppose that $e \leq e^{\prime}$. Then:

1. Either $K=K^{\prime}$ or $K^{\prime}=F K$.

2. Suppose $E$ has potential supersingular reduction. Let $e_{1}$ and $e_{1}^{\prime}$ be the valuation of the coefficient of $X^{p}$ in the power series $[p](X)$ for the formal groups $\widehat{E}$ and $\widehat{E^{\prime}}$ respectively, as defined above, and assume that $e_{1}<e$. Then,

$$
e^{\prime}=\mu e, \text { and } e_{1}^{\prime}=\mu e_{1},
$$

where $\mu=\left[K^{\prime}: K\right]=1$ or 2 .

Proof. Since $E$ and $E^{\prime}$ are isomorphic over $F$, it follows that they are also isomorphic over $F K$. Since $E$ has good reduction over $K$, it also has good reduction over $F K$ (see [12], VII, Proposition 5.4, part (b)). Thus, $E^{\prime}$ has good reduction over $F K$ as well. By the properties of $K^{\prime}$ (see our comments at the beginning of Section 2) we know that $K^{\prime} \subseteq F K$. Similarly, $K \subseteq F K^{\prime}$. In particular, $F K K^{\prime}=F K=F K^{\prime}$.

Suppose that $K \neq K^{\prime}$ and $e<e^{\prime}$. Notice that

$$
K \subsetneq K K^{\prime} \subseteq F K K^{\prime}=F K .
$$

Thus, $K K^{\prime} / K$ is quadratic, and so is $K^{\prime} / K \cap K^{\prime}$. It follows that $K^{\prime} / K \cap K^{\prime}$ is a non-trivial (tamely) ramified quadratic extension of $K \cap K^{\prime}$ and, since $p \geq 3$ and $L_{\wp}^{\mathrm{nr}} \subseteq K \cap K^{\prime}$, there is a unique such extension of $K \cap K^{\prime}$. Since we have assumed that $K \neq K^{\prime}$, it follows that $K / K \cap K^{\prime}$ is trivial, $K \subseteq K^{\prime}$, and $K^{\prime} / K=K^{\prime} / K \cap K^{\prime}$ is quadratic. Since $K \subsetneq K^{\prime} \subseteq K K^{\prime} \subseteq$ $F K$, and $F K / K$ is at most quadratic, it follows that $K^{\prime}=F K$, as desired. This proves (1).

For (2), let $H=F K=F K^{\prime}$, and assume that $e \leq e^{\prime}$, as before. By part (1), either $K=K^{\prime}$ and $e=e^{\prime}$, or $K^{\prime}=F K$ is quadratic over $K$ and $e^{\prime}=2 e$. Thus, $e^{\prime}=\mu \cdot e$ with $\mu=\left[K^{\prime}: K\right]$. Now, the formulas in Theorem 3.3 applied to $E$ say that $e_{1}=\lambda=e / e(\wp \mid p) \cdot C$, where 
$C=C(j(E), \wp)$ is a constant that only depends on $j(E)$ and $\wp$. Let us apply Theorem 3.3 to $E^{\prime} / A^{\prime}$ :

$$
\lambda^{\prime}=e^{\prime} / e(\wp \mid p) \cdot C\left(j\left(E^{\prime}\right), \wp\right)=\mu e / e(\wp \mid p) \cdot C(j(E), \wp)=\mu \lambda,
$$

where we have used the fact that $j(E)=j\left(E^{\prime}\right)$ because $E \cong_{F} E^{\prime}$. Since $\lambda=e_{1}<e$ by assumption, it follows that $\lambda^{\prime}=\mu \lambda<\mu e=e^{\prime}$. Hence, the theorem implies that $e_{1}^{\prime}=\lambda^{\prime}=\mu \lambda=\mu e_{1}$, as claimed.

Lemma 4.11. Let $F$ be a field of characteristic 0 , and let $E / F$ and $E^{\prime} / F$ be isomorphic elliptic curves (over a fixed algebraic closure $\bar{F}$ ) with $j(E)=j\left(E^{\prime}\right) \neq 0$ or 1728. Let $\phi: E \rightarrow$ $E^{\prime}$ be an isomorphism. Then:

1. $E$ and $E^{\prime}$ are isomorphic over $F$ or $E^{\prime}$ is a quadratic twist of $E$.

2. For all $R \in E(\bar{F})$, we have $F(x(R))=F(x(\phi(R)))$.

Proof. Let $E$ and $E^{\prime}$, respectively, be given by Weierstrass equations $y^{2}=x^{3}+A x+B$ and $y^{2}=x^{3}+A^{\prime} x+B^{\prime}$, with coefficients in $F$. Since $j(E)=j\left(E^{\prime}\right) \neq 0,1728$, none of the coefficients is zero. By [12], Ch. III, Prop. 3.1(b), the isomorphism $\phi: E \rightarrow E^{\prime}$ is given by $(x, y) \mapsto\left(u^{2} x, u^{3} y\right)$ for some $u \in \bar{F} \backslash\{0\}$. Hence $A^{\prime}=u^{4} A$ and $B^{\prime}=u^{6} B$, and so $u^{2} \in F$. Thus, either $E \cong_{F} E^{\prime}$, or $E^{\prime}$ is the quadratic twist of $E$ by $u$. This shows (1).

Let $R \in E(\bar{F})$. If $E \cong_{\mathbb{Q}} E^{\prime}$ then $F(R)=F(\phi(R))$ and the same holds for the subfields of the $x$-coordinates, so (2) is immediate. Let us assume for the rest of the proof that $E^{\prime}$ is the quadratic twist of $E$ by $\sqrt{d}$, for some $d \in F \backslash F^{2}$. It follows that $\phi((x, y))=(d x, d \sqrt{d} \cdot y)$ and, therefore, $F(x(\phi(R)))=F(d \cdot x(R))=F(x(R))$. This proves (2).

\section{Formal groups and the valuation of torsion points}

In this section we apply our previous results about the formal group of an elliptic curve with potential supersingular reduction to calculate the slopes in the Newton polygon of the multiplication-by- $p$ map. In turn, the slopes will allow us to calculate the valuation of $p^{n}$-th torsion points in the formal group, and the ramification index in the extensions generated by these points.

Lemma 5.1. Let $E, K$ and $v$ be as above, so that $E / K$ is an elliptic curve given by a minimal model with good supersingular reduction. Put $[p](X)=\sum_{i=1}^{\infty} s_{i} X^{i}$ and let $e=$ $v\left(s_{1}\right)=v(p)$ and $e_{1}=v\left(s_{p}\right)$. Let $T_{1} \in E(\bar{K})[p]$ be a non-trivial $p$-torsion point, fix any sequence $\left\{T_{n} \in E\left[p^{n}\right]:[p] T_{n+1}=T_{n}\right\}$, and let $t_{n}$ be the corresponding torsion points in $\widehat{E}(\mathcal{M})$, where $\mathcal{M}$ is the maximal ideal in the ring of integers of $\bar{K}$.

(i) $\quad v\left(t_{n+1}\right)<v\left(t_{n}\right)$;

(ii) If $v\left(t_{n}\right)<\frac{e p}{p-1}$, then $v\left(t_{n+1}\right)<\frac{v\left(t_{n}\right)}{p}$;

(iii) If $v\left(t_{m}\right)<\min \left\{e, e_{1}\right\}$ for some $m \geq 1$, then for all $n \geq m$ we have

$$
v\left(t_{n}\right)=\frac{v\left(t_{m}\right)}{p^{2(n-m)}} \text {. }
$$

In particular, the ramification index of $K\left(T_{n+1}\right) / K\left(T_{n}\right)$ is $p^{2}$ for all $n \geq m$.

Proof. The theory of formal groups (see [12], VII, Proposition 2.2) shows that there is an isomorphism $t: E_{1}(\bar{K}) \cong \widehat{E}(\mathcal{M})$, where $\widehat{E}$ is the formal group associated to $E$. The 
isomorphism is given by $(x, y) \mapsto t((x, y))=-x / y$. Since we are assuming that $E / K$ has good supersingular reduction, all torsion points with $p$-power order live in the kernel of reduction $E_{1}(\bar{K})$. Thus, $T_{n} \in E(\bar{K})\left[p^{n}\right] \subset E_{1}$, for $n=1,2, \ldots$, and we define $t_{n}=t\left(T_{n}\right)$.

Since $E / K$ is good supersingular, the height of $\widehat{E}$ as a formal group is 2. As above, there are power series $f(X), g(X)$ and $h(X)$ in $X \cdot A[[X]]$, with $f^{\prime}(0)=g^{\prime}(0)=h^{\prime}(0) \in A^{\times}$, such that:

$$
[p](X)=p f(X)+\pi^{e_{1}} g\left(X^{p}\right)+h\left(X^{p^{2}}\right) .
$$

It follows that

$$
\begin{aligned}
v\left(t_{n}\right)=v\left([p]\left(t_{n+1}\right)\right) & \geq \min \left\{v\left(p f\left(t_{n+1}\right)\right), v\left(\pi^{e_{1}} g\left(t_{n+1}^{p}\right)\right), v\left(h\left(t_{n+1}^{p^{2}}\right)\right)\right\} \\
& =\min \left\{e+v\left(t_{n+1}\right), e_{1}+p v\left(t_{n+1}\right), p^{2} v\left(t_{n+1}\right)\right\}>v\left(t_{n+1}\right) .
\end{aligned}
$$

It follows that $v\left(t_{n+1}\right)<v\left(t_{n}\right)$ as claimed in (i). Assume that $v\left(t_{n}\right)<e p /(p-1)$ or, equivalently, $e+v\left(t_{n}\right) / p>v\left(t_{n}\right)$. For a contradiction, suppose that $v\left(t_{n+1}\right) \geq v\left(t_{n}\right) / p$. By Eq. (2), this would imply $v\left(t_{n}\right) \geq \min \left\{e+v\left(t_{n}\right) / p, e_{1}+v\left(t_{n}\right), p v\left(t_{n}\right)\right\}>v\left(t_{n}\right)$, a contradiction. This proves (ii).

We will prove (iii) using induction. Clearly, the base case $n=m$ is trivial. Now, suppose the equality is valid for some $n>m$, i.e., $v\left(t_{n}\right)=v\left(t_{m}\right) / p^{2(n-m)}$. In particular, $v\left(t_{n}\right)<v\left(t_{m}\right)<\min \left\{e, e_{1}\right\}$. Hence, the only possibility in Eq. (2) is that the minimum is attained with $p^{2} v\left(t_{n+1}\right)$, and since this value is smaller than the other two, all inequalities are actually equalities. Thus, $v\left(t_{n}\right)=p^{2} v\left(t_{n+1}\right)$. Hence,

$$
v\left(t_{n+1}\right)=\frac{v\left(t_{n}\right)}{p^{2}}=\frac{v\left(t_{m}\right)}{p^{2(n-m)+2}}=\frac{v\left(t_{m}\right)}{p^{2(n+1-m)}} .
$$

Thus, by the principle of mathematical induction, the equality is valid for all $n \geq m$.

Lemma 5.2. Let $E / L$ be an elliptic curve with potential good supersingular reduction at a prime $\wp$. Let $K_{E} / L_{\wp}^{n r}, A, \pi$, e, and $e_{1}$ be as above, so that $[p](X)=p f(X)+\pi^{e_{1}} g\left(X^{p}\right)+$ $h\left(X^{p^{2}}\right)$, where $f(X), g(X)$ and $h(X)$ are power series in $X \cdot A[[X]]$, with $f^{\prime}(0)=g^{\prime}(0)=$ $h^{\prime}(0) \in A^{\times}$. Then:

1. If pe $/(p+1) \leq e_{1}$, then $[p](X)=0$ has $p^{2}-1$ roots of valuation $\frac{e}{p^{2}-1}$;

2. If $p e /(p+1)>e_{1}$, then $[p](X)=0$ has $p-1$ roots with valuation $\frac{e-e_{1}}{p-1}$ and $p^{2}-p$ roots with valuation $\frac{e_{1}}{p(p-1)}$.

Proof. This is shown in [10], $\S 1.10$ (pp. 271-272). Let $N$ be the part of the Newton polygon of $[p](Z)$ that describes the roots of valuation $>0$. Let $P_{0}=(1, e), P_{1}=\left(p, e_{1}\right)$, and $P_{2}=\left(p^{2}, 0\right)$. The slope of the segment $P_{0} P_{1}$ is $-\left(e-e_{1}\right) /(p-1)$, while the slope of the segment $P_{0} P_{2}$ is $-e /\left(p^{2}-1\right)$. It follows from the theory of Newton polygons (see [10], p. 272) that:

1. If $p e /(p+1) \leq e_{1}$, then $N$ is given by a single segment $P_{0} P_{2}$ of length $p^{2}-1$. Thus, there are $p^{2}-1$ roots of valuation $\frac{e}{p^{2}-1}$.

2. Otherwise, if $p e /(p+1)>e_{1}$, then $N$ is given by two segments $P_{0} P_{1}$ and $P_{1} P_{2}$ of length $p-1$ and $p^{2}-p$, respectively. It follows that there are $p-1$ roots with valuation $\frac{e-e_{1}}{p-1}$ and $p^{2}-p$ roots with valuation $\frac{e_{1}}{p(p-1)}$. 
We say that $t$ is a primitive root of $\left[p^{n}\right](X)=0$ if $\left[p^{n}\right](t)=0$ but $\left[p^{m}\right](t) \neq 0$ for any $0 \leq m<n$.

Lemma 5.3. With notation as in Lemma 5.2, let $n \geq 1$ be fixed.

1. If pe $/(p+1) \leq e_{1}$, then every primitive root of $\left[p^{n}\right](X)=0$ has valuation $\frac{e}{p^{2(n-1)}\left(p^{2}-1\right)}$;

2. If pe $/(p+1)>e_{1}$, then $\left[p^{n}\right](X)=0$ has $\left(p^{2}-p\right) p^{2(n-1)}$ primitive roots with valuation $\frac{e_{1}}{(p-1) p^{2 n-1}}$. Moreover, let $s$ be the smallest non-negative integer such that $e / e_{1} \leq p^{s}(p+1)$. Then,

(a) if $n \leq s+1$, there are $(p-1) p^{2(n-1)}$ primitive roots of valuation $\frac{e-p^{n-1} e_{1}}{(p-1) p^{n-1}}$;

(b) if $n>s+1$, there are $(p-1) p^{2(n-1)}$ primitive roots of valuation $\frac{e-p^{s} e_{1}}{(p-1) p^{2(n-1)-s}}$.

Proof. Let $t_{n}$ be a primitive root of $\left[p^{n}\right](X)=0$. Then, $t_{1}=\left[p^{n-1}\right](X)$ is a non-zero root of $[p](X)=0$. By Lemma 5.2 there are three options according to the valuation of $t_{1}$.

1. If $p e /(p+1) \leq e_{1}$, then the valuation of $t_{1} \in \widehat{E}[p]$ is $e /\left(p^{2}-1\right)$. Thus,

$$
v\left(t_{1}\right)=\frac{e}{p^{2}-1}<p(p-1) \frac{e}{p^{2}-1}=\frac{p e}{p+1} \leq \min \left\{e, e_{1}\right\} .
$$

Hence, by Lemma 5.1,

$$
v\left(t_{n}\right)=\frac{v\left(t_{1}\right)}{p^{2(n-1)}}=\frac{e}{p^{2(n-1)}\left(p^{2}-1\right)} .
$$

2. If $p e /(p+1)>e_{1}$, then the valuation of $t_{1}$ is $e_{1} / p(p-1)$ or $\left(e-e_{1}\right) /(p-1)$.

Notice that in this case $e>p e /(p+1)>e_{1}$. If $v\left(t_{1}\right)=e_{1} / p(p-1) \leq \min \left\{e, e_{1}\right\}$, then by Lemma 5.1,

$$
v\left(t_{n}\right)=\frac{v\left(t_{1}\right)}{p^{2(n-1)}}=\frac{e_{1}}{p^{2(n-1)+1}(p-1)}=\frac{e_{1}}{p^{2 n-1}(p-1)} .
$$

For the rest of the proof, let us assume that $v\left(t_{1}\right)=\left(e-e_{1}\right) /(p-1)$. We note that $v\left(t_{1}\right)<e$. Let us write $t_{m}=\left[p^{n-m}\right]\left(t_{n}\right)$. In the proof of Lemma 5.1 we saw that

$$
v\left(t_{m}\right) \geq \min \left\{e+v\left(t_{m+1}\right), e_{1}+p v\left(t_{m+1}\right), p^{2} v\left(t_{m+1}\right)\right\}>v\left(t_{m+1}\right),
$$

for any $1 \leq m<n$. Since $v\left(t_{m}\right)>v\left(t_{m+1}\right)$ for all $m$, and $v\left(t_{1}\right)<e$, it follows that the minimum cannot be $e+v\left(t_{m+1}\right)$ for any $m \geq 1$. Thus,

$$
v\left(t_{m}\right) \geq \min \left\{e_{1}+p v\left(t_{m+1}\right), p^{2} v\left(t_{m+1}\right)\right\},
$$

and the inequality is an equality, unless $e_{1}+p v\left(t_{m+1}\right)=p^{2} v\left(t_{m+1}\right)$, i.e., $v\left(t_{m+1}\right)=e_{1} /\left(p^{2}-p\right)$. Thus, there are three options,

$$
v\left(t_{m+1}\right)=\frac{v\left(t_{m}\right)-e_{1}}{p}, \text { or } \frac{v\left(t_{m}\right)}{p^{2}}, \text { or } \frac{e_{1}}{p^{2}-p},
$$

according to whether the minimum is attained at $e_{1}+p v\left(t_{m+1}\right)$, at $p^{2} v\left(t_{m+1}\right)$, or at $e_{1}+p v\left(t_{m+1}\right)=p^{2} v\left(t_{m+1}\right)$, respectively. The first option happens when $v\left(t_{m+1}\right)>e_{1} /\left(p^{2}-p\right)$, and the second option when $v\left(t_{m+1}\right)<e_{1} /\left(p^{2}-p\right)$. Equivalently, $v\left(t_{m+1}\right)=\left(v\left(t_{m}\right)-e_{1}\right) / p$ if $v\left(t_{m}\right)>p e_{1} /(p-1)$, and $v\left(t_{m+1}\right)=v\left(t_{m}\right) / p^{2}$ if $v\left(t_{m}\right) \leq p e_{1} /(p-1)$. Let $s$ be the smallest non-negative integer such that $e / e_{1} \leq p^{s}(p+1)$. 
(a) We shall prove by induction that $v\left(t_{n}\right)=\frac{e-p^{n-1} e_{1}}{(p-1) p^{n-1}}$ for all $1 \leq n \leq s+1$.

The base case of $n=1$ follows from our assumtion that

$v\left(t_{1}\right)=\left(e-e_{1}\right) /(p-1)$. Now suppose that $1 \leq n<s+1$, and

$v\left(t_{n}\right)=\frac{e-p^{n-1} e_{1}}{(p-1) p^{n-1}}$. Since $n-1<s$, it follows that $e>p^{n-1}(p+1) e_{1}$. Thus,

$v\left(t_{n}\right)=\frac{e-p^{n-1} e_{1}}{(p-1) p^{n-1}}>\frac{p^{n-1}(p+1) e_{1}-p^{n-1} e_{1}}{(p-1) p^{n-1}}=\frac{p^{n-1} p e_{1}}{(p-1) p^{n-1}}=\frac{p e_{1}}{p-1}$.

By our previous remarks, this inequality implies that

$$
v\left(t_{n+1}\right)=\frac{v\left(t_{n}\right)-e_{1}}{p}=\frac{\frac{e-p^{n-1} e_{1}}{(p-1) p^{n-1}}-e_{1}}{p}=\frac{e-p^{n} e_{1}}{(p-1) p^{n}} .
$$

Thus, by the principle of mathematical induction, the result follows for all $1 \leq n \leq s+1$.

(b) Here we shall show by induction that $v\left(t_{n}\right)=\frac{e-p^{s} e_{1}}{(p-1) p^{2(n-1)-s}}$ for all $n \geq s+1$. The previous case shows that $v\left(t_{s+1}\right)=\frac{e-p^{s} e_{1}}{(p-1) p^{s}}$. Since $e \leq p^{s}(p+1) e_{1}$, it follows that

$$
v\left(t_{s+1}\right)=\frac{e-p^{s} e_{1}}{(p-1) p^{s}} \leq \frac{p^{s}(p+1) e_{1}-p^{s} e_{1}}{(p-1) p^{s}}=\frac{p^{s} p e_{1}}{(p-1) p^{s}}=\frac{p e_{1}}{p-1} .
$$

By our previous remarks, this inequality implies that

$$
v\left(t_{s+2}\right)=\frac{v\left(t_{s+1}\right)}{p^{2}}=\frac{e-p^{s} e_{1}}{(p-1) p^{s+2}}=\frac{e-p^{s} e_{1}}{(p-1) p^{2(s+2-1)-s}} .
$$

Now suppose that $n>s+1$ and $v\left(t_{n}\right)=\frac{e-p^{s} e_{1}}{(p-1) p^{2(n-1)-s}}$. Since $n>s+1$, it follows that $v\left(t_{n}\right)<v\left(t_{s+1}\right) \leq p e_{1} /(p-1)$. Therefore, our previous remarks show that

$$
v\left(t_{n+1}\right)=\frac{v\left(t_{n}\right)}{p^{2}}=\frac{e-p^{s} e_{1}}{(p-1) p^{2(n-1)-s+2}}=\frac{e-p^{s} e_{1}}{(p-1) p^{2((n+1)-1)-s}},
$$

as desired. Hence, the principle of mathematical induction shows that

$$
v\left(t_{n}\right)=\frac{e-p^{s} e_{1}}{(p-1) p^{2(n-1)-s}}
$$

for all $n \geq s+1$.

Example 5.4. Let $E / \mathbb{Q}$ be the elliptic curve with Cremona label "27a4", given by a Weierstrass equation $y^{2}+y=x^{3}-30 x+63$. The curve $E$ has additive reduction at $p=3$, which turns out to be potential good supersingular reduction. In this case, the good reduction is first attained over a number field $K_{0}=\mathbb{Q}(\alpha, \beta)$, where $\alpha$ and $\beta$ are roots of the polynomials $x^{4}-3$ and $x^{3}-120 x+506$, respectively. The extension $K_{0} / \mathbb{Q}$ is of degree 12 , totally ramified at $p=3$. We define $K=K_{0} \mathbb{Q}_{3}^{\text {nr }}$. In this particular case, we have $e=12$, and we have also calculated $e_{1}=2$.

We have calculated (using Magma) the coordinates of torsion points $T_{1}, T_{2}$ and $T_{3}$ in $E^{\prime}$, respectively of order 3,9 and 27 , such that [3] $T_{3}=T_{2}$, and [3] $T_{2}=T_{1}$. There are two non-trivial 3-torsion points defined over $K$ (this follows from the fact that $E$ has a 3-torsion point defined over $\mathbb{Q}$ ), and we let $T_{1}$ be one of them. Let $F_{3} / \mathbb{Q}$ and $F_{9} / \mathbb{Q}$ be 
unique extensions of degrees 3 and 9 contained in $\mathbb{Q}\left(\zeta_{27}\right) / \mathbb{Q}$. Then $T_{2} \in E^{\prime}\left(K F_{3}\right)$ and $T_{3} \in E^{\prime}\left(K F_{9}\right)$. If we let $t_{i}=-x\left(T_{i}\right) / y\left(T_{i}\right)$, we find that

$$
v\left(t_{1}\right)=5, \quad v\left(t_{2}\right)=1, \quad \text { and } \quad v\left(t_{3}\right)=1 / 9 .
$$

Notice that $3 \cdot 12 / 4=9>2=e_{1}$, thus by Lemma 5.3 , the formal group has $6 \cdot 3^{2(n-1)}$ primitive roots with valuation $\frac{2}{2 \cdot 3^{2 n-1}}=\frac{1}{3^{2 n-1}}$. Moreover, $12 / 2=6 \leq 3 \cdot 4$, so $s=1$. Hence,

1. if $n \leq 2$, there are $2 \cdot 3^{2(n-1)}$ primitive roots of valuation $\frac{12-3^{n-1} \cdot 2}{2 \cdot 3^{n-1}}$;

2. if $n>2$, there are $2 \cdot 3^{2(n-1)}$ primitive roots of valuation

$$
\frac{12-3 \cdot 2}{2 \cdot 3^{2(n-1)-1}}=\frac{1}{3^{2(n-1)-2}}=\frac{1}{3^{2 n-4}} \text {. }
$$

In particular, there are precisely two points of 3-torsion in the formal group with valuation $\left(e-e_{1}\right) /(p-1)=(12-2) / 2=5$ and $t_{1}$ is one of them (the rest of the 3 -torsion points, 6 of them, have valuation 1/3). Also, there are 18 points of 9 -torsion in the formal group with valuation 1 , and $t_{2}$ is one of them (the other 54 torsion points of order 9 have valuation $1 / 27$ ). Finally, there are 162 roots of 27 -torsion with valuation $1 / 9$ and $t_{3}$ is one of them (the other 486 roots of order 27 have valuation $1 / 243$ ).

Example 5.5. Let $E=E_{121 \mathrm{c} 2}$ defined over $\mathbb{Q}$. As we know $e=3$ and $e_{1}=1$ for $p=11$. Since $33 / 12>1$, it follows from Lemma 5.3 that $\left[11^{n}\right](X)=0$ has $110 \cdot 11^{2(n-1)}$ primitive roots with valuation $\frac{1}{10 \cdot 11^{2 n-1}}$, for all $n \geq 1$. Moreover, $e / e_{1}=3 \leq 12$ implies that $s=0$, and so for all $n \geq 1$, there are $10 \cdot 11^{2(n-1)}$ primitive roots of valuation $\frac{1}{5 \cdot 11^{2(n-1)}}$. In particular, when $n=1$, there are 110 roots with valuation $1 / 110$, and 10 roots with $1 / 5$.

Proposition 5.6. Let $E / L$ be an elliptic curve with potential good supersingular reduction at a prime $p$. Let $K$ be the smallest extension of $L_{\wp}^{n r}$ such that $E / K$ has good (supersingular) reduction at $p$, and let $e=v(p)$ and $e_{1}=v\left(s_{p}\right)$ be defined as above.

1. If $p e /(p+1) \leq e_{1}$, then the ramification index in the extension $K\left(T_{n}\right) / K$ is divisible by $\left(p^{2}-1\right) p^{2(n-1)} / \operatorname{gcd}\left(e,\left(p^{2}-1\right) p^{2(n-1)}\right)$, where $T_{n} \in E\left[p^{n}\right]$ is an arbitrary torsion point on $E$ of exact order $p^{n}$.

2. If pe $/(p+1)>e_{1}$, then there are $\left(p^{2}-p\right) p^{2(n-1)}$ torsion points $T_{n} \in E\left[p^{n}\right]$ such that the ramification index in $K\left(T_{n}\right) / K$ is divisible by $(p-1) p^{2 n-1} / \operatorname{gcd}\left(e_{1},(p-1) p^{2 n-1}\right)$. Moreover, let $s$ be the smallest non-negative integer such that $e / e_{1} \leq p^{s}(p+1)$. Then,

(a) if $n \leq s+1$, there are $(p-1) p^{2(n-1)}$ points $T_{n} \in E\left[p^{n}\right]$ such that the ramification index in $K\left(T_{n}\right) / K$ is divisible by $(p-1) p^{n-1} / \operatorname{gcd}\left(e-p^{n-1} e_{1}\right.$, $\left.(p-1) p^{n-1}\right)$;

(b) if $n>s+1$, there are $(p-1) p^{2(n-1)}$ points $T_{n} \in E\left[p^{n}\right]$ such that the ramification index in $K\left(T_{n}\right) / K$ is divisible by $(p-1) p^{2(n-1)-s} / \operatorname{gcd}\left(e-p^{s} e_{1}\right.$, $\left.(p-1) p^{2(n-1)-s}\right)$.

In all cases, there is a number $c=c(E / L, T, \wp)$ with $1 \leq c \leq e \leq 24 e(\wp \mid p)$ such that if $T \in E\left[p^{n}\right]$ is of order $p^{n}$, then the ramification index in $K(T) / K$ is divisible by $\varphi\left(p^{n}\right) / \operatorname{gcd}\left(c, \varphi\left(p^{n}\right)\right)$. 
Proof. Let $T_{n} \in E\left[p^{n}\right]$ be an arbitrary point on $E(\bar{K})$ of exact order $p^{n}$, and write $T_{i}=\left[p^{n-i}\right] T_{n}$, for $i=1, \ldots, n$. Also, write $t_{i}$ for the corresponding torsion point in the formal group, i.e., $t_{i}=t\left(T_{i}\right)=-x\left(T_{i}\right) / y\left(T_{i}\right) \in \widehat{E}(\mathcal{M})$. The proposition now follows from Lemma 5.3. In the last statement, we simply pick $c=e, e_{1}, e-p^{n-1} e_{1}$, or $e-p^{s} e_{1}$. Notice that if $p e /(p+1)>e_{1}$, then $e>e_{1}$. Thus, in all cases, $1 \leq c \leq e$.

The previous proposition has the following asymptotic consequence for the growth of ramification indices.

Corollary 5.7. Let E/L be an elliptic curve with potential good supersingular reduction at a prime $p$. Let $K$ be the smallest extension of $L_{\wp}^{n r}$ such that $E / K$ has good (supersingular) reduction at $p$. Let $\left\{T_{n} \in E\left[p^{n}\right]\right\}_{n=1}^{\infty}$ be an arbitrary sequence of torsion points, such that $T_{n}$ has exact order $p^{n}$. Then, there is an integer $m=m(E / K) \geq 1$ such that

$$
e\left(K\left(T_{n+1}\right) / K\right)=e\left(K\left(T_{n}\right) / K\right) \cdot p^{2}
$$

for all $n \geq m$.

Proof. The statement follows directly from Proposition 5.6, by letting

$$
m=\max \left\{s+1,\left\lceil\left(v_{p}(e)+2\right) / 2\right\rceil,\left\lceil\left(v_{p}\left(e-p^{s} e_{1}\right)+s+2\right) / 2\right\rceil\right\}
$$

where $\lceil\cdot\rceil$ is the integer ceiling function.

Remark 5.8. Let $L$ be a number field with ring of integers $\mathcal{O}_{L}$, and let $\wp$ be a prime ideal of $\mathcal{O}_{L}$ lying above a rational prime $p$. Let $E / L$ be an elliptic curve, and let $R \in E(\bar{L})\left[p^{n}\right]$ be a point of exact order $p^{n}$. Let $\iota: \bar{L} \hookrightarrow \bar{L}_{\wp}$ be a fixed embedding. Let $F=L(R)$ and let $\Omega_{R}$ be the prime of F above $\wp$ associated to the embedding $\iota$. Let $K$ be a finite Galois extension of $L_{\wp}^{n r}$, such that the ramification index of $K$ over $\mathbb{Q}_{p}$ is e. Let $\tilde{E} / K$ be a curve isomorphic to $E$ over $K$, and let $T \in \tilde{E}(K)\left[p^{n}\right]$ be the point that corresponds to $(R)$ on $E\left(\bar{L}_{\wp}\right)$. Suppose that the degree of the extension $K(T) / K$ is $g$. Since $K / L_{\wp}^{n r}$ is of degree e/e $(\wp \mid p)$, it follows that the degree of $K(T) / L_{\wp}^{n r}$ is eg/e(§|p).

Let $\mathcal{F}=\iota(F) \subseteq \bar{L}_{\wp}$. Since $E$ and $\tilde{E}$ are isomorphic over $K$, it follows that $K(T)=K \mathcal{F}$ and, therefore, the degree of the extension $K \mathcal{F} / L_{\wp}^{n r}$ is eg/e $(\wp \mid p)$. Since $K / L_{\wp}^{n r}$ is Galois by assumption, it follows that $g=[K(T): K]=\left[\mathcal{F} L_{\wp}^{n r}: K \cap \mathcal{F} L_{\wp}^{n r}\right]$, so the degree of $\left[\mathcal{F} L_{\wp}^{n r}: L_{\wp}^{n r}\right]$ equals $g \cdot k$ where $k=\left[K \cap \mathcal{F} L_{\wp}^{n r}: L_{\wp}^{n r}\right]$. Hence, the degree of $\mathcal{F} / L_{\wp}$ is divisible by gk and, in particular, the ramification index of the prime ideal $\Omega_{R}$ over $\wp$ in the extension $L(R) / L$ is divisible by $g k$, where $g=[K(T): K]$.

Theorem 5.9. Let $\eta \geq 1$ and $n \geq 1$ be fixed. Let $p$ be a prime, let $L$ be a number field, and let $\wp$ be a prime ideal of $\mathcal{O}_{L}$ lying above $p$, such that $e(\wp \mid p) \leq \eta$. Let $E / L$ be an elliptic curve with potential supersingular reduction at $\wp$, let $R \in E\left[p^{n}\right]$ be a point of exact order $p^{n}$. Then, there is a number $c=c(E / L, R, \wp)$ with $1 \leq c \leq 24 \eta$ (with $c \leq 12 \eta$ if $p>2$, and $c \leq 12 \eta$ if $p>3)$, such that the ramification index $e(\mathfrak{P} \mid \wp)$ of any prime $\mathfrak{P}$ above $\wp$ in the extension $L(R) / L$ is divisible by $\varphi\left(p^{n}\right) / \operatorname{gcd}\left(c, \varphi\left(p^{n}\right)\right)$. Moreover, 
1. There is a constant $f(\eta)$, which depends only on $\eta$, such that $c \mid f(\eta)$. Moreover $f(\eta)$ is a divisor of $F(\eta)=\operatorname{lcm}(\{n: 1 \leq n<24 \eta, \operatorname{gcd}(n, 6) \neq 1\})$. If $p>3$, then $f(\eta)$ is a divisor of $F_{0}(\eta)=\operatorname{lcm}(\{n: 1 \leq n<6 \eta, \operatorname{gcd}(n, 6) \neq 1\})$.

2. Let $\sigma$ be the smallest non-negative integer such that $8 \eta \leq 2^{\sigma}$ (or such that $\eta \leq 5^{\sigma}$, if $p>3)$. If $n>\sigma+1$, then $e(\mathfrak{P} \mid \wp)$ is divisible by $(p-1) p^{2(n-1)-\sigma} / \operatorname{gcd}((p-1)$ $\left.p^{2(n-1)-\sigma}, c\right)$.

3. If $p>3 \eta$, then $e(\mathfrak{P} \mid \wp)$ is divisible by $(p-1) p^{n-1} / \operatorname{gcd}(p-1, c)$.

4. If $\eta=1$ and $p>3$, then $e(\mathfrak{P} \mid \wp)$ is divisible by $\left(p^{2}-1\right) p^{2(n-1)} / 6$, or $(p-1) p^{2(n-1)} / \operatorname{gcd}(p-1,4)$. If $\eta=1$ and $p=3$, then $e(\mathfrak{P} \mid \wp)$ is divisible by $\varphi\left(3^{n}\right) / \operatorname{gcd}\left(\varphi\left(3^{n}\right), t\right)$ with $t=6$ or 9 .

Proof. Since $F(\eta)$ is a divisor of $F\left(\eta^{\prime}\right)$ (respectively, $F(\eta)$ is a divisor of $F_{0}\left(\eta^{\prime}\right)$ ) whenever $\eta \leq \eta^{\prime}$, it suffices to show the theorem when $e(\wp \mid p)=\eta$. By Proposition 5.6 and Remark 5.8 it follows that $e(\mathfrak{P} \mid \wp)$ is divisible by one of the following quantities:

$$
\begin{aligned}
& \frac{\left(p^{2}-1\right) p^{2(n-1)}}{\operatorname{gcd}\left(\left(p^{2}-1\right) p^{2(n-1)}, e\right)}, \quad \text { or } \quad \frac{(p-1) p^{2 n-1}}{\operatorname{gcd}\left((p-1) p^{2 n-1}, e_{1}\right)}, \\
& \text { or } \frac{(p-1) p^{n-1}}{\operatorname{gcd}\left((p-1) p^{n-1}, e-p^{n-1} e_{1}\right)} \quad \text { if } n \leq s+1, \\
& \text { or } \frac{(p-1) p^{2(n-1)-s}}{\operatorname{gcd}\left((p-1) p^{2(n-1)-s}, e-p^{s} e_{1}\right)} \quad \text { if } n>s+1,
\end{aligned}
$$

where $s$ is the smallest non-negative integer such that $e / e_{1} \leq p^{s}(p+1)$, so we define $c=e$, $e_{1}, e-p^{n-1} e_{1}$, or $e-p^{s} e_{1}$ accordingly, and it follows that $1 \leq c \leq e$, so the bounds on $c$ follow from the discussion on the possible values of $e$ at the beginning of Section 2. Hence, in all cases $e(\mathfrak{P} \mid \wp)$ is divisible by $(p-1) p^{n-1} / \operatorname{gcd}\left((p-1) p^{n-1}, c\right)$.

By Corollary 4.5 , there is a constant $f(\eta, p)$ such that $e, e_{1}, e-p^{n-1} e_{1}$, and $e-p^{s} e_{1}$ are divisors of $f(\eta, p)$, so $c \mid f(\eta, p)$ as well. Moreover, $f(\eta, p)$ divides $F(\eta)$, and $f(\eta, p)$ divides $F_{0}(\eta)$ for $p>3$. Hence, in all cases $e(\mathfrak{P} \mid \wp)$ is divisible by $\varphi\left(p^{n}\right) / \operatorname{gcd}\left(\varphi\left(p^{n}\right), F(\eta)\right)$. This shows (1).

Let $\sigma$ be the smallest non-negative integer such that $8 \eta \leq 2^{\sigma}$ (or such that $\eta \leq 5^{\sigma}$, if $p>3)$. Since $e=e / e(\wp \mid p) \cdot e(\wp \mid p)$, and $e^{\prime}=e / e(\wp \mid p)$ is $\leq 24$ (resp. $\leq 6$, if $\left.p>3\right)$, then

$$
e / e_{1} \leq 24 \eta \leq 3 \cdot 2^{\sigma} \leq p^{\sigma}(p+1)
$$

(resp. $e / e_{1} \leq 12 \eta \leq 6 \cdot 5^{\sigma} \leq p^{\sigma}(p+1)$ if $\left.p \geq 5\right)$. Since $s$ is smallest such that $p^{s}(p+1) \geq$ $e / e_{1}$, it follows that $s \leq \sigma$. Hence, if $n>\sigma+1$, then $n>s+1$ and $e(\mathfrak{P} \mid \wp)$ is divisible by one of

$$
\frac{\left(p^{2}-1\right) p^{2(n-1)}}{\operatorname{gcd}\left(\left(p^{2}-1\right) p^{2(n-1)}, c\right)} \text {, or } \quad \frac{(p-1) p^{2 n-1}}{\operatorname{gcd}\left((p-1) p^{2 n-1}, c\right)}, \quad \text { or } \quad \frac{(p-1) p^{2(n-1)-s}}{\operatorname{gcd}\left((p-1) p^{2(n-1)-s}, c\right)} \text {, }
$$

or with $c$ replaced by $F(\eta)$. Hence, in all cases $e(\mathfrak{P} \mid \wp)$ is divisible by the quantity

$$
(p-1) p^{2(n-1)-s} / \operatorname{gcd}\left((p-1) p^{2(n-1)-s}, c\right) \text {. }
$$

This shows (2).

By Corollary 4.8, if $p>3 \eta \geq 3 e(\wp \mid p)$, then $e, e_{1}, e-p^{n-1} e_{1}$, and $e-p^{s} e_{1}$ are not divisible by $p$. It follows that $e(\mathfrak{P} \mid \wp)$ is divisible by $(p-1) p^{n-1} / \operatorname{gcd}(p-1, c)$, and it is divisible by $(p-1) p^{2(n-1)-s} / \operatorname{gcd}(p-1, c)$ if $n>\sigma+1$, and with $c$ replaced by $F(\eta)$. This shows (3).

If $\eta=e(\wp \mid p) \leq 1$ and $p>3$, Corollary 4.5 says that $e$ divides 4 or 6 , and $e_{1}, e-e_{1}$, are 
divisors of 4 (note that $e / e_{1} \leq p+1$ for all $p>3$, so $s=0$ ). Hence, $e(\mathfrak{P} \mid \wp)$ is divisible by one of

$$
\frac{\left(p^{2}-1\right) p^{2(n-1)}}{\operatorname{gcd}\left(p^{2}-1,6\right)} \text {, or } \frac{\left(p^{2}-1\right) p^{2(n-1)}}{\operatorname{gcd}\left(p^{2}-1,4\right)} \text {, or } \frac{(p-1) p^{2 n-1}}{\operatorname{gcd}(p-1,4)} \text {, or } \frac{(p-1) p^{2(n-1)}}{\operatorname{gcd}(p-1,4)} \text {. }
$$

It follows that $e(\mathfrak{P} \mid \wp)$ is divisible by $\left(p^{2}-1\right) p^{2(n-1)} / 6$ or $(p-1) p^{2(n-1)} / \operatorname{gcd}(p-1,4)$. If $d=1$ and $p=3$, then $e(\mathfrak{P} \mid \wp)$ is divisible by $\varphi\left(3^{n}\right) / \operatorname{gcd}\left(\varphi\left(3^{n}\right), c\right)$ where $1 \leq c \leq 12$. Since $\varphi\left(3^{n}\right)=2 \cdot 3^{n-1}$, it is also divisible by $\varphi\left(3^{n}\right) / \operatorname{gcd}\left(\varphi\left(3^{n}\right), t\right)$ with $t=6$ or 9 . This shows (4) and concludes the proof of the theorem.

Example 5.10. Let $E / \mathbb{Q}$ be the elliptic curve with Cremona label "27a4", given by a Weierstrass equation $y^{2}+y=x^{3}-30 x+63$. We have seen in Example 5.4 that for this curve and $p=3$, we have $e=12$ and $e_{1}=2$. In particular $p e /(p+1)=3 \cdot 12 / 4=9>2$ and Proposition 5.6 implies that, for all $n \geq 1$, there are $6 \cdot 3^{2 n-2}$ points $T_{n}$ in $E\left(\overline{\mathbb{Q}}_{3}\right)\left[3^{n}\right]$ such that the ramification index in $K\left(T_{n}\right) / K$ is divisible by $2 \cdot 3^{2 n-1} /\left(\operatorname{gcd}\left(3^{2 n-1} \cdot 2,2\right)\right)=$ $3^{2 n-1}$.

Let $T_{i} \in E\left[3^{i}\right]$ for $i=1,2,3$ be the torsion points defined in Example 5.4. We also defined $t_{i}=-x\left(T_{i}\right) / y\left(T_{i}\right)$, and we found that $v\left(t_{1}\right)=5, v\left(t_{2}\right)=1$, and $\quad v\left(t_{3}\right)=1 / 9$. Since $v\left(t_{3}\right)<\min \{12,2\}=2$, Lemma 5.1 implies that for all $n \geq 3$, and all $T_{n} \in E\left[3^{n}\right]$ such that $\left[3^{n-3}\right]\left(T_{n}\right)=T_{3}$ we have $v\left(t_{n}\right)=\frac{v\left(t_{3}\right)}{3^{2(n-3)}}=\frac{1}{3^{2 n-4}}$. Thus, the ramification index of $K\left(T_{n}\right) / K$ is divisible by $3^{2 n-4}$, when $n \geq 3$.

In all cases, we find that, if $T_{n}$ is any point of exact order $3^{n}$ and $n \geq 3$, then the ramification index of $K\left(T_{n}\right) / K$ is divisible either $3^{2 n-1}$ or by $3^{2 n-4}$, so it is divisible by, at least, $3^{2 n-4}$. Thus, Remark 5.8 implies that if $R \in E(\overline{\mathbb{Q}})$ is a point of exact order $3^{n}$ with $n \geq 3$, then the ramification index of any prime lying above 3 in the extension $\mathbb{Q}(R) / \mathbb{Q}$ is divisible by $3^{2 n-4}$.

Example 5.11. Let $E=E_{121 \mathrm{c} 2}$ defined over $\mathbb{Q}$. As we know $e=3$ and $e_{1}=1$ for $p=11$. Since $33 / 12>1$, by Proposition 5.6 , there are $110 \cdot 11^{2(n-1)}$ torsion points $T_{n} \in E\left[p^{n}\right]$ such that $c\left(E / \mathbb{Q}, T_{n}, 11\right)=e_{1}=1$ and, therefore, the ramification index in $\mathbb{Q}\left(T_{n}\right) / \mathbb{Q}$ is divisible by $10 \cdot 11^{2 n-1}$. Moreover, $e / e_{1}=3 \leq 12$, so $s=0$, and there are $10 \cdot 11^{2(n-1)}$ points $T_{n} \in E\left[p^{n}\right]$ such that $c\left(E / \mathbb{Q}, T_{n}, 11\right)=e-e_{1}=2$, and the ramification index in $\mathbb{Q}\left(T_{n}\right) / \mathbb{Q}$ is divisible by $5 \cdot 11^{2(n-1)}$ for all $n \geq 1$. In particular, if $T_{n}$ is any point of order $11^{n}$, then the ramification index at 11 in $\mathbb{Q}\left(T_{n}\right) / \mathbb{Q}$ is divisible by $5 \cdot 11^{2(n-1)}$ for all $n \geq 1$.

Let $F=\mathbb{Q}\left(\zeta_{11}\right)$ be the 11th cyclotomic field, and let $\zeta=\zeta_{11}$ be a primitive 11 th root of unity. Then, $E$ has a point of exact order 11 defined over $F$, namely

$$
\begin{aligned}
T_{1}= & \left(-22 \zeta^{9}-11 \zeta^{7}-11 \zeta^{6}-11 \zeta^{5}-11 \zeta^{4}-22 \zeta^{2}+17\right. \\
& \left.-77 \zeta^{9}-33 \zeta^{8}-132 \zeta^{7}-33 \zeta^{6}-33 \zeta^{5}-132 \zeta^{4}-33 \zeta^{3}-77 \zeta^{2}-201\right) .
\end{aligned}
$$

Moreover, notice that both $x\left(T_{1}\right)$ and $y\left(T_{1}\right)$ are fixed by complex conjugation and, therefore, $T_{1}$ is defined over $F^{+}=\mathbb{Q}\left(\zeta_{11}\right)^{+}$, the maximal real subfield of $F$. Moreover, one can verify that $\mathbb{Q}\left(T_{1}\right)=F^{+}$. Thus, the ramification index of $11 \mathrm{in} \mathbb{Q}\left(T_{1}\right) / \mathbb{Q}$ is 5 , which is the smallest it can be, since it must be divisible by $5 \cdot 11^{2(n-1)}$ with $n=1$.

We finish this section with a result on the behavior of ramification under quadratic twists. 
Proposition 5.12. Let $E / L$ be an elliptic curve with potential supersingular reduction at a prime ideal $\wp$ above $p \geq 3$, and let $T_{n} \in E\left[p^{n}\right]$ be a point of exact order $p^{n}$. Let $K$ be the smallest extension of $L_{\wp}^{n r}$ such that $E / K$ has good reduction, let $v$ be a normalized valuation on $K$, and let $e=v(p)$ and $e_{1}=v\left(s_{p}\right)$ defined as usual. Let $E^{\prime} / L$ be a quadratic twist of $E$, such that $E$ and $E$ are isomorphic over a quadratic extension $F / L$, and let $K^{\prime}, v^{\prime}$, $e^{\prime}$, and $e_{1}^{\prime}$ be the analogous items attached to $E^{\prime}$.

1. If $F / L$ is unramified at $\wp$, then $e=e^{\prime}$ and $e_{1}=e_{1}^{\prime}$, and the results of Proposition 5.6 apply equally to $E$ or $E^{\prime}$.

2. Otherwise, assume that $F / L$ is ramified at $\wp$. If $K / L_{\wp}^{\mathrm{nr}}$ contains a quadratic ramified extension, then $e=e^{\prime}$ and $e_{1}=e_{1}^{\prime}$, and the results of Proposition 5.6 apply equally to $E$ or $E^{\prime}$.

3. Finally, assume that $F / L$ is ramified at $\wp$, and assume further that $L\left(x\left(T_{n}\right)\right)$ contains a quadratic extension $H / L$ ramified at $\wp$. Let $T_{n}^{\prime} \in E^{\prime}\left[p^{n}\right]$ be the point on $E^{\prime}$ that corresponds to $T_{n}$ on $E\left[p^{n}\right]$. Then, $L\left(T_{n}\right) / L$ and $L\left(T_{n}^{\prime}\right) / L$ have the same ramification properties for primes that lie above $\wp$.

Proof. Part (1) is clear, since $F K=K$. For part (2), let $K_{0} / L_{\wp}^{\mathrm{nr}}$ be the quadratic extension contained in $K / L_{\wp}^{\mathrm{nr}}$. Then $F L_{\wp}^{\mathrm{nr}}=K_{0}$. Thus, $F K=K$ and, by Lemma 4.10, we have $K=$ $K^{\prime}$, and the result follows. Finally, for (3), we have $L\left(x\left(T_{n}\right)\right)=L\left(x\left(T_{n}^{\prime}\right)\right)$ by Lemma 4.11. Let us fix an embedding $\iota: \bar{L} \hookrightarrow \bar{L}_{\wp}$ and put $\mathcal{F}_{n}=\iota\left(L\left(T_{n}\right)\right)$ and $\mathcal{F}_{n}^{\prime}=\iota\left(L\left(T_{n}^{\prime}\right)\right)$. Since $p \geq 3$, it follows that $F L_{\wp}^{\mathrm{nr}}=H L_{\wp}^{\mathrm{nr}}$, therefore $F L_{\wp}^{\mathrm{nr}} \subseteq \mathcal{F}_{n}$, and $F L_{\wp}^{\mathrm{nr}} \subseteq \mathcal{F}_{n}^{\prime}$. Since $E \cong_{F} E^{\prime}$, it follows that

$$
\mathcal{F}_{n} L_{\wp}^{\mathrm{nr}}=\iota\left(L\left(T_{n}\right)\right) F L_{\wp}^{\mathrm{nr}}=\iota\left(L\left(T_{n}^{\prime}\right)\right) F L_{\wp}^{\mathrm{nr}}=\mathcal{F}_{n}^{\prime} L_{\wp}^{\mathrm{nr}},
$$

and the result follows.

\section{Examples from $X_{0}\left(p^{n}\right)$}

In this last section, we discuss examples of elliptic curves with potential supersingular reduction that appear associated to non-cuspidal rational points on a modular curve $X_{0}\left(p^{n}\right)$ for some prime $p$ and $n \geq 1$.

Let $E / \mathbb{Q}$ be an elliptic curve with a $\mathbb{Q}$-rational cyclic isogeny $\phi$ of degree $p^{n}$. Then, the pair $(E, C)$ with $C=\operatorname{Ker}(\phi)$ corresponds to a $\mathbb{Q}$-rational point on the modular curve $X_{0}\left(p^{n}\right)$. Conversely, following [1], each non-cuspidal $\mathbb{Q}$-rational point on $X_{0}\left(p^{n}\right)$ comes from such a pair $(E / \mathbb{Q},\langle R\rangle)$, with $R \in E\left[p^{n}\right]$. The rational points on the modular curves $X_{0}\left(p^{n}\right)$ have been completely classified (see, for example, Section 9.1 and Tables 2, 3, and 4 of [5]). Here, in Table 1, we list every non-cuspidal $\mathbb{Q}$-rational point on the modular curves $X_{0}\left(p^{n}\right)$ of genus $\geq 1$, which correspond to elliptic curves with potential supersingular reduction at the prime $p$ (and provide the Cremona labels for curves with the given $j$ invariant and least conductor). We remark here that $X_{0}(27), X_{0}(11), X_{0}(17)$, and $X_{0}(19)$ have genus 1 , but only contain finitely many $\mathbb{Q}$-rational points.

Theorem 6.1. Let $\left(j_{0}, p\right)$ be any of the j-invariants that are listed in Table 1, together with the corresponding prime pof potential supersingular reduction. Let $E / \mathbb{Q}$ be an elliptic 
Table 1 Elliptic curves with pot. supersingular reduction on $X_{0}\left(p^{n}\right)$ of genus $\geq 1$

\begin{tabular}{|c|c|c|c|c|c|c|}
\hline j-invariant & $p$ & $n$ & Cremona label(s) & Good reduction over & $e$ & $\overline{e_{1}}$ \\
\hline$j=-2^{15} \cdot 3 \cdot 5^{3}$ & 3 & 3 & $27 a 2,27 a 4$ & $\mathbb{Q}\left(\sqrt[4]{3}, \beta^{3}-120 \beta+506=0\right)$ & 12 & $\overline{2}$ \\
\hline$j=-11 \cdot 131^{3}$ & \multirow{3}{*}{11} & \multirow{3}{*}{1} & $121 c 2$ & $\mathbb{Q}(\sqrt[3]{11})$ & 3 & 1 \\
\hline$j=-2^{15}$ & & & $121 b 1,121 b 2$ & $\mathbb{Q}(\sqrt[4]{11})$ & 4 & 2 \\
\hline$j=-11^{2}$ & & & $121 \mathrm{c} 1$ & $\mathbb{Q}(\sqrt[3]{11})$ & 3 & 2 \\
\hline$j=-17^{2} \cdot 101^{3} / 2$ & \multirow{2}{*}{17} & \multirow{2}{*}{1} & 14450p1 & $\mathbb{Q}(\sqrt[3]{17})$ & 3 & 2 \\
\hline$j=-17 \cdot 373^{3} / 2^{17}$ & & & 14450p2 & $\mathbb{Q}(\sqrt[3]{17})$ & 3 & 1 \\
\hline$j=-2^{15} \cdot 3^{3}$ & 19 & 1 & $361 \mathrm{a} 1,361 \mathrm{a} 2$ & $\mathbb{Q}(\sqrt[4]{19})$ & 4 & 2 \\
\hline$j=-2^{18} \cdot 3^{3} \cdot 5^{3}$ & 43 & 1 & 1849a1, 1849a2 & $\mathbb{Q}(\sqrt[4]{43})$ & 4 & 2 \\
\hline$j=-2^{15} \cdot 3^{3} \cdot 5^{3} \cdot 11^{3}$ & 67 & 1 & 4489a1, 4489a2 & $\mathbb{Q}(\sqrt[4]{67})$ & 4 & 2 \\
\hline$j=-2^{18} \cdot 3^{3} \cdot 5^{3} \cdot 23^{3} \cdot 29^{3}$ & 163 & 1 & $26569 a 1,26569 a 2$ & $\mathbb{Q}(\sqrt[4]{163})$ & 4 & 2 \\
\hline
\end{tabular}

curve with $j(E)=j_{0}$, and let $T_{n} \in E\left[p^{n}\right]$ be a point of exact order $p^{n}$. Then, the ramification index of any prime $\wp$ that lies above $p$ in the extension $\mathbb{Q}\left(T_{n}\right) / \mathbb{Q}$ is divisible by $(p-1) p^{2 n-2} / 2$ if $p>3$ and $n \geq 1$, and by $3^{2 n-4}$ if $p=3$ and $n \geq 3$.

Proof. With the notation of the statement of the theorem, fix a prime $\Omega_{\wp}$ of $\overline{\mathbb{Q}}$ that lies above $\wp$, and let $\iota_{\wp}: \overline{\mathbb{Q}} \hookrightarrow \overline{\mathbb{Q}}_{p}$ be the embedding associated to $\Omega_{\wp}$. We divide the $j$-invariants in three subsets:

- Let $j_{0}=-2^{15} \cdot 3 \cdot 5^{3}$ and $p=3$. Let $E / \mathbb{Q}$ be the elliptic curve with Cremona label "27a4". Then, we have worked out in Example 5.10 that, for all $n \geq 3$, the ramification index of $\mathbb{Q}_{3}\left(\iota\left(T_{n}\right)\right) / \mathbb{Q}_{3}$ is divisible by $3^{2 n-4}$. Hence, the ramification of $\wp$ over $p$ in the extension $\mathbb{Q}\left(T_{n}\right) / \mathbb{Q}$ is also divisible by $3^{2 n-4}$. Since the smallest field extension of $\mathbb{Q}_{3}^{\mathrm{nr}}$ such that $E$ acquires good reduction is given by $K=\mathbb{Q}_{3}^{\mathrm{nr}}\left(\sqrt[4]{3}, \beta^{3}-120 \beta+506=0\right)$ (see Table 1 ), it follows that $K / \mathbb{Q}_{3}^{\text {nr }}$ contains the quadratic extension $\mathbb{Q}_{3}^{\mathrm{nr}}(\sqrt{3}) / \mathbb{Q}_{3}^{\mathrm{nr}}$, and therefore, by Proposition 5.12, parts (1) and (2), any quadratic twist of $E / \mathbb{Q}$ shares the same ramification properties in the extension $\mathbb{Q}\left(T_{n}\right) / \mathbb{Q}$. Since any elliptic curve over $\mathbb{Q}$ with $j=j(E)$ is a quadratic twist of $E / \mathbb{Q}$ (by Lemma 4.11), we are done.

- Let $j_{0}$ be one of the $j$-invariants with $p=11,19,43,67$ or 163 , and let $E / \mathbb{Q}$ be one of the elliptic curves with Cremona label as listed in Table 1. From the same table, we see that in all cases $e-e_{1}$ and $e_{1}$ are 1 or 2 . If $E / \mathbb{Q}$ is replaced by a quadratic twist, then Lemma 4.10 says that the quantities $\left(e, e_{1}\right)$ stay the same or are replaced by ( $2 e, 2 e_{1}$ ), and $2 e-2 e_{1}$ and $2 e_{1}$ are 2 or 4 . (Notice that, in fact, in [4], Cor. 4.6, we have shown that if $E / L$ has potential supersingular reduction at $\wp$, with $e(\wp \mid p)=1$, and $e_{1}<e$, then $e-e_{1}$ and $e_{1}$ can only take the values 1,2 , or 4$)$. Moreover, in all cases $p \equiv 3 \bmod 4$, so $\operatorname{gcd}(p(p-1), 4)=\operatorname{gcd}(p(p-1), 4)=2$. Also, in all cases it can be easily verified that $p e /(p+1)>e_{1}$ and, equivalently, $p(2 e) /(p+1)>2 e_{1}$. Therefore, Proposition 5.6 implies that the ramification index in the extension $K\left(\iota\left(T_{n}\right)\right) / K$ is divisible by $(p-1) p^{2 n-2} / 2$. Hence, by Remark 5.8 , the ramification index of $\wp$ over $p$ in the extension $\mathbb{Q}\left(T_{n}\right) / \mathbb{Q}$ is divisible by $(p-1) p^{2 n-2} / 2$ for any elliptic curve with $j=j_{0}$.

- Let $j_{0}$ be one of the two $j$-invariants with $p=17$. Let $E / \mathbb{Q}$ be the elliptic curve with Cremona label "14450p1". Let $T_{n}$ be a point of exact order $17^{n}$ on $E$. We claim that 
$\mathbb{Q}\left(x\left(T_{n}\right)\right)$ contains $\mathbb{Q}(\sqrt{17})$. First notice that, if $T_{1}=\left[p^{n-1}\right] T_{n} \in E[17]$, then $\mathbb{Q}\left(x\left(T_{1}\right)\right) \subseteq \mathbb{Q}\left(x\left(T_{n}\right)\right)$ because the function $f=x \circ\left[17^{n-1}\right]$ is even, and therefore lies in the function field $\mathbb{Q}(x)$ (see [12], Cor. 2.3.1). The $x$-coordinate of $T_{1}$ is a root of $\psi_{17}(x)$, the 17 th division polynomial of $E$. The division polynomial factors as $\psi_{17}(x)=s_{1}(x) s_{2}(x)$, where $s_{1}(x)$ and $s_{2}(x)$ have degrees 8 and 144 respectively. Let $\alpha_{i}$ be a root of $s_{i}(x)$, for $i=1,2$. We have verified with the software Magma that $\mathbb{Q}(\sqrt{17}) \subseteq \mathbb{Q}\left(\alpha_{i}\right)$ for both $i=1$ and 2 . Therefore, $\mathbb{Q}(\sqrt{17}) \subseteq \mathbb{Q}\left(x\left(T_{1}\right)\right) \subseteq \mathbb{Q}\left(x\left(T_{n}\right)\right)$. Similarly, if we let $E^{\prime} / \mathbb{Q}$ be the curve with label "14450p2", the 17th division polynomial factors as $\psi_{17}(x)=s_{1}(x) s_{2}(x) s_{3}(x)$ where the polynomials $s_{i}$ have degrees 4,4 and 136, respectively for $i=1,2$, and 3 . Let $\alpha_{i}$ be a root of $s_{i}(x)$. We have also verified with the software Magma that $\mathbb{Q}(\sqrt{17}) \subseteq \mathbb{Q}\left(\alpha_{i}\right)$ for both $i=1,2$, and 3 . Therefore, $\mathbb{Q}(\sqrt{17}) \subseteq \mathbb{Q}\left(x\left(T_{1}\right)\right) \subseteq \mathbb{Q}\left(x\left(T_{n}\right)\right)$, for any $T_{n} \in E^{\prime}$ of order $17^{n}$. In particular, if $T_{n} \in E$ or $E^{\prime}$, parts (1) and (3) of our Proposition 5.12 imply that the ramification properties at $p$ of $\mathbb{Q}\left(T_{n}\right)$ are invariant under quadratic twists, and therefore it suffices to show the theorem for $E$ and $E^{\prime}$. From Table 1 we see that, for $E$ we have $\left(e-e_{1}, e_{1}\right)=(1,2)$, and for $E^{\prime}$ we have $\left(e-e_{1}, e_{1}\right)=(2,1)$. Hence, $\operatorname{gcd}\left(17 \cdot 16, e-e_{1}\right)$ and $\operatorname{gcd}\left(17 \cdot 16, e_{1}\right)$ are both $\leq 2$. Moreover, in both cases $p e /(p+1)>e_{1}$ for $p=17$. Hence, by Proposition 5.6 and Remark 5.8, we have that the ramification index of $\wp$ over $(17)$ in the extension $\mathbb{Q}\left(T_{n}\right) / \mathbb{Q}$ is divisible by $16 \cdot 17^{2 n-2} / 2=8 \cdot 17^{2 n-2}$, for all $n \geq 1$, as desired.

We conclude the paper with an example of an elliptic curve defined over a quadratic number field $L$, which appears as a non-cuspidal $L$-point on $X_{0}(13)(L)$.

Example 6.2. Let $j_{0}$ be a root of the polynomial

$$
x^{2}-6896880000 x-567663552000000,
$$

and let $L=\mathbb{Q}\left(j_{0}\right)=\mathbb{Q}(\sqrt{13})$. Let $p=13$ and let $\wp=(\sqrt{13})$ be the ideal above $p$ in $\mathcal{O}_{L}$. Let $E / L$ be the elliptic curve with $j$-invariant equal to $j_{0}$. The curve $E$ has complex multiplication by $\mathbb{Z}[\sqrt{-13}]$, i.e., $\operatorname{End}(E / \mathbb{C}) \cong \mathbb{Z}[\sqrt{-13}]$ and, in fact, all the endomorphisms are defined over $\mathbb{Q}(\sqrt{13}, i)$, see [13], Chapter 2, Theorem 2.2(b)). Since 13 ramifies in $L$, it follows from Deuring's criterion (see [3], Ch. 13, §4, Theorem 12) that the reduction of $E$ at $\wp$ is potential supersingular. We choose a model for $E / L$ given by

$$
y^{2}=x^{3}+\frac{5231 j_{0}-50692880808000}{3825792} x+\frac{-550711 j_{0}+4485396184200000}{239112} .
$$

The discriminant of this model is $\Delta_{L}=\frac{13546495176890000 j_{0}-93429639900045292464000000}{29889}$ and $v_{\wp}\left(\Delta_{L}\right)=0$. Hence, $E / L$ has good supersingular reduction at $\wp$. In particular $K_{E}=L_{\wp}^{\mathrm{nr}}$ and $e=2$. Since $p=13 \equiv 1 \bmod 12$, we have $r(13)=s(13)=0$, and we may use Theorem 3.3 to verify that $e_{1}=1$. Here $e(\wp \mid p)=2$, and we know from Example 3.2 that $Q_{13}(T)=-349920 T-75582720$. One can verify (using Sage or Magma) that

$$
v_{\wp}\left(Q_{13}\left(j_{0}\right)\right)=v_{\wp}\left(-349920 j_{0}-75582720\right)=1 .
$$

Thus,

$$
\lambda=v_{K}\left(Q_{13}(j(E))=v_{\wp}\left(Q_{13}\left(j_{0}\right)\right)=1 .\right.
$$


Since $1=\lambda<2=e$, it follows from Theorem 3.3 that $e_{1}=\lambda=1$, as claimed.

Since $26 / 14>1$ and $e_{1}=1$, Proposition 5.6 and Remark 5.8 imply that there are $156 \cdot 13^{2(n-1)}$ torsion points $T_{n} \in E\left[p^{n}\right]$ such that the ramification index in $L\left(T_{n}\right) / L$ is divisible by $12 \cdot 13^{2 n-1}$. Moreover, $2 \leq 14$ so $s=0$, and $e-e_{1}=1$. Thus, there are $12 \cdot 13^{2(n-1)}$ points $T_{n} \in E\left[p^{n}\right]$ such that the ramification index in $L\left(T_{n}\right) / L$ is divisible by $12 \cdot 13^{2(n-1)}$.

\section{Acknowledgements}

I would like to thank Kevin Buzzard, Brian Conrad, Harris Daniels, and Felipe Voloch for several useful references and suggestions.

Received: 18 August 2015 Accepted: 16 February 2016

Published online: 15 April 2016

References

1. Deligne, P, Rapoport, M: Les schémas de modules des courbes elliptiques, in Modular Functions of One Variable II. Springer Lecture Notes Math. 349, 143-316 (1973)

2. Flexor, M, Oesterlé, J: Sur les points de torsion des courbes elliptiques. Astérisque. 183, 25-36 (1990)

3. Lang, S: Elliptic functions. Second Edition. Springer-Verlag, New Tork (1987)

4. Lozano-Robledo, Á: Formal groups of elliptic curves with potential good supersingular reduction. Pac. J. Math. 261(1), 145-164 (2013)

5. Lozano-Robledo, Á: On the field of definition of $p$-torsion points on elliptic curves over the rationals. Math. Annalen. 357(1), 279-305 (2013)

6. Lozano-Robledo, Á: Uniform boundedness in terms of ramification. preprint

7. Merel, L: Bornes pour la torsion des courbes elliptiques sur les corps de nombres. Invent. Math. 124(1-3), 437-449 (1996)

8. Néron, A: Modèles minimaux des variétés abéliennes sur les corps locaux et globaux. Inst. Hautes Etudes Sci. Publ. Math. 21, 128 (1964)

9. Parent, P: Bornes effectives pour la torsion des courbes elliptiques sur les corps de nombres. J. Reine Angew. Math. 506, 85-116 (1999)

10. Serre, J-P: Propriétés galoisiennes des points d'ordre fini des courbes elliptiques. Invent. Math. 15, $259-331$ (1972)

11. Serre, J-P, Tate, J: Good reduction of abelian varieties. Ann. Math. 88, 492-517 (1968)

12. Silverman, JH: The Arithmetic of Elliptic Curves. 2nd Edition. Springer-Verlag, New York (2009)

13. Silverman, JH: Advanced Topics in the Arithmetic of Elliptic Curves. Springer-Verlag, New York (1994)

\section{Submit your manuscript to a SpringerOpen ${ }^{\circ}$ journal and benefit from:}

- Convenient online submission

- Rigorous peer review

- Immediate publication on acceptance

- Open access: articles freely available online

- High visibility within the field

- Retaining the copyright to your article

Submit your next manuscript at $\mathbf{s p r i n g e r o p e n . c o m ~}$ 